hep-th/0011075

\title{
Type IIA and Heterotic String Vacua in $D=2 \rrbracket$
}

\author{
Michael Haack ${ }^{a}$ ], Jan Louis ${ }^{a / 3}$ and Monika Marquart ${ }^{a, b \text { f }}$ \\ ${ }^{a}$ Fachbereich Physik, Martin-Luther-Universiät Halle-Wittenberg, \\ Friedemann-Bach-Platz 6, D-06099 Halle, Germany \\ ${ }^{b}$ Department of Physics, Queen Mary and Westfield College \\ Mile End Road, London E1, U.K.
}

\begin{abstract}
We study type IIA string theory compactified on Calabi-Yau fourfolds and heterotic string theory compactified on Calabi-Yau threefolds times a two-torus. We derive the resulting effective theories which have two space-time dimensions and preserve four supercharges. The duality between such vacua is established at the level of the effective theory. For type IIA vacua with non-trivial Ramond-Ramond background fluxes a superpotential is generated. We show that for a specific choice of background fluxes and a fourfold which has the structure of a threefold fibred over a sphere the superpotential coincides with the superpotential recently proposed by Taylor and Vafa in compactifications of type IIB string theory on a threefold.
\end{abstract}

November 2000

\footnotetext{
${ }^{1}$ Work supported by: DFG - The German Science Foundation, GIF - the German-Israeli Foundation for Scientific Research, DAAD - the German Academic Exchange Service and the Landesgraduiertenförderung Sachsen-Anhalt.

${ }^{2}$ email: michael@hera1.physik.uni-halle.de

${ }^{3}$ email: j.louis@physik.uni-halle.de

${ }^{4}$ email: marquart@hera1.physik.uni-halle.de
} 


\section{Introduction}

Among the various string theory vacua those with four supercharges in $D=4$ spacetime dimensions are phenomenologically the most relevant ones. Such vacua have been intensively studied and in string perturbation theory they are constructed for example by compactification of the heterotic string on a Calabi-Yau threefold $Y_{3}$ [1]. Some of the non-perturbative properties of such vacua can be obtained from a dual F-theory compactification on an appropriate Calabi-Yau fourfold $Y_{4}$ [2].

Sometimes the non-perturbative features of a given string vacuum simplify in lower dimensions as a consequence of a simpler dual description. For example the dual of the heterotic string compactified on $Y_{3} \times S^{1}$ is given by M-theory compactified on $Y_{4}$ while the dual of the heterotic string compactified on $Y_{3} \times T^{2}$ is type IIA compactified on $Y_{4}$ [2].

In this paper we focus on string vacua in $D=2$ with four supercharges, the heterotic string compactified on $Y_{3} \times T^{2}$ and the dual type IIA compactified on $Y_{4}$. In the first part of the paper (section 2) we derive the low energy effective theories and establish the duality at the level of the effective theory. We display an explicit map between the heterotic variables and their dual type IIA counterparts. In the second part of the paper (section 3) we consider type IIA backgrounds where non-trivial RR-fluxes have been turned on. The specific case of a 4 -form flux can be used to construct a consistent compactification if the Euler number of the fourfold is non-vanishing [3]. The presence of such fluxes leads to a non-trivial superpotential $W$ and a supersymmetric vacuum is found only on a subspace of the moduli space «, 5, 6, 7, 8, 9, 10].

The superpotential receives two distinct contributions $W$ and $\tilde{W}$ [7, 9]. $\tilde{W}$ arises from wrapping $\mathrm{D} p$-branes on holomorphic $p$-cycles and depends on the deformations of the (complexified) Kähler class, i.e. the harmonic (1,1)-forms on $Y_{4}$. $W$ on the other hand is generated by wrapped $D 4$-branes on special Lagrangian cycles and thus depends on the deformations of the complex structure or the harmonic $(1,3)$-forms on $Y_{4}$. $\tilde{W}$ receives quantum corrections on the worldsheet whereas $W$ is determined by a classical computation. Mirror symmetry relates the two superpotentials and demands $\tilde{W}\left(Y_{4}\right)=$ $W\left(\tilde{Y}_{4}\right)$, where $\tilde{Y}_{4}$ is the mirror fourfold of $Y_{4}$.

In section 3.2 we show that for a particular class of fourfolds which have the structure of a Calabi-Yau threefold $Y_{3}$ fibred over a base $\mathbb{P}_{1}$ the superpotential is determined by the prepotential $\mathcal{F}$ of $Y_{3}$ if the background fluxes are suitably chosen and the $\mathbb{P}_{1}$ is taken to be large. Curiously this superpotential coincides with the superpotential derived in ref. [11]. It arises in type II compactification on a Calabi-Yau threefold with non-vanishing fluxes studied in refs. [12, 13, 11, 14]. It also is very closely related to the BPS-mass formula studied in refs. [15, 16] and the entropy of $N=2$ extremal black holes studied in refs. [17, 18.

In section 3.3 we consider fourfolds which have the structure of a $K 3$ manifold fibred over a (complex) two-dimensional Hirzebruch surface $\mathbf{F}_{n}$. In the limit of a large base $\mathbf{F}_{n}$ the superpotential is determined by the prepotential of $K 3$ fibred threefolds in the large base limit. In this case the duality to heterotic vacua discussed in section 2.3 can be used to also determine the heterotic superpotential.

Finally, some of the technical details are deferred to three appendices. 


\section{Heterotic - type IIA duality in $D=2$ without su- perpotential}

\subsection{Heterotic vacua in $D=2$}

In this section we perform a Kaluza-Klein reduction of a generic $D=4, N=1$ heterotic effective theory on a torus $T^{2}$. This results in an effective theory in $D=2$ with $(2,2)$ supersymmetry. For the purpose of this paper it is sufficient to focus only on the vector multiplets and the chiral moduli multiplets in the $D=4$ effective action and ignore all charged matter multiplets. The bosonic part of the effective Lagrangian in this case reads

$$
\mathcal{L}_{\text {het }}^{(4)}=\frac{1}{2} R^{(4)}-G_{I \bar{J}}^{(4)}(\Phi, \bar{\Phi}) \partial_{m} \Phi^{I} \partial^{m} \bar{\Phi}^{\bar{J}}-\frac{1}{4} \operatorname{Re} f_{a b}(\Phi) F_{m n}^{a} F^{b m n}+\frac{1}{4} \operatorname{Im} f_{a b}(\Phi) F_{m n}^{a} \tilde{F}^{b m n}
$$

where $m, n=0, \ldots, 3, \Phi^{I}$ are the moduli fields and $F_{m n}^{a}$ is the field strength of the gauge bosons $A_{m}^{a}, a=1, \ldots, \operatorname{dim}(G)$. The $f_{a b}(\Phi)$ are holomorphic functions of the moduli and the metric $G_{I \bar{J}}^{(4)}$ is a Kähler metric with Kähler potential $K^{(4)}(\Phi, \bar{\Phi})$. At the string tree level $K^{(4)}$ and $f_{a b}$ are further constrained to obey

$$
K_{\text {het }}^{(4)}(\phi, \bar{\phi}, S, \bar{S})=\tilde{K}_{\text {het }}^{(4)}(\phi, \bar{\phi})-\ln [i(\bar{S}-S)], \quad f_{a b}=-i S \delta_{a b}
$$

where $S$ is the heterotic dilaton and the $\phi$ denote all moduli except the dilaton.f

The next step is to perform a Kaluza-Klein reduction on a torus. The chiral multiplets survive the reduction unaltered and continue to contain a complex scalar field as bosonic component. The vectors on the other hand decompose according to

$$
A_{n}^{a} d x^{n}=A^{a} d \zeta+\bar{A}^{a} d \bar{\zeta}+A_{\mu}^{a} d x^{\mu}
$$

where $\mu=0,1$ and $\zeta$ is the complex coordinate on the torus. The vectors $A_{\mu}^{a}$ do not have any dynamical degrees of freedom and play the role of auxiliary fields. Thus the physical bosonic components of the vector multiplets in $D=2$ consist of the complex scalars $A^{a}$. These scalars transform in the adjoint representation of the gauge group $G$ and thus their vacuum expectation values break $G$ to its maximal Abelian subgroup $U(1)^{\operatorname{rank}(G)}$. With a slight abuse of notation the index $a$ from now on only takes the values $a=1, \ldots, \operatorname{rank}(G)$. On this Coulomb branch each vector occurs in the Lagrangian only via its Abelian field strength. In this case the field strength can be replaced by an auxiliary scalar field [19] and one ends up with the field content of a twisted chiral multiplet [20]. This is one of the two different kinds of matter multiplets which can occur in $D=2$ theories with $(2,2)$ supersymmetry, the other one being the chiral multiplet. We later use the fact that under certain conditions they are dual to each other. More precisely, if the Lagrangian is invariant under a Peccei-Quinn shift symmetry of a chiral multiplet one can perform a duality transformation replacing a chiral by a twisted chiral multiplet - and vice versa [20].

\footnotetext{
${ }^{5}$ For the general case see appendix $\mathrm{B}$.

${ }^{6}$ Contrary to the standard heterotic convention we choose to identify the dilaton with $\operatorname{Im} S$ in order to simplify the discussion in section 3 .
} 
Finally, we need to decompose the four-dimensional space-time metric. Instead of using the Einstein metric implicit in eq. (1) it turns out to be more convenient to work in the string frame metric and decompose it according to 7

$$
g_{m n} d x^{m} d x^{n}=g_{\mu \nu}^{(2)} d x^{\mu} d x^{\nu}+b_{\mu i} d x^{\mu} d x^{i}+h_{i j} d x^{i} d x^{j}, \quad i, j=2,3,
$$

where $g_{\mu \nu}^{(2)}$ and $b_{\mu i}$ have no physical degrees of freedom. The real coordinates $x^{2}, x^{3}$ are related to the complex $\zeta$ of eq. (3) via $d \zeta=d x^{2}+\tau d x^{3}$ where $\tau$ is the complex structure modulus of the torus

$$
\tau \equiv\left(\frac{h_{12}}{h_{11}}+i \frac{\sqrt{h}}{h_{11}}\right)
$$

and $h$ is the determinant of $h_{i j}$.

We omit the details of the standard Kaluza-Klein compactification on the torus and only present the resulting two-dimensional effective Lagrangian

$$
\begin{aligned}
\mathcal{L}_{\text {het }}^{(2)} & =e^{-2 \Phi_{\text {het }}^{(2)}}\left[-\frac{1}{2} \partial_{\mu} \partial^{\mu} \sigma+\frac{\partial_{\mu} \tau \partial^{\mu} \bar{\tau}}{(\tau-\bar{\tau})^{2}}-\frac{1}{4} h^{-1} \partial_{\mu} \sqrt{h} \partial^{\mu} \sqrt{h}-\tilde{G}_{I \bar{J}}^{(4)} \partial_{\mu} \phi^{I} \partial^{\mu} \bar{\phi}^{\bar{J}}\right. \\
& \left.-i(\tau-\bar{\tau})^{-1} \sqrt{h}^{-1} D_{\mu} \bar{n}^{a} D^{\mu} n^{a}-h^{-1}\left(\partial_{\mu} P+\frac{1}{2}(\tau-\bar{\tau})^{-1}\left[\bar{n}^{a} D_{\mu} n^{a}-n^{a} D_{\mu} \bar{n}^{a}\right]\right)^{2}\right],
\end{aligned}
$$

where we defined

$$
e^{-2 \Phi_{\mathrm{het}}^{(2)}}=\frac{\sqrt{h}}{2 i}(S-\bar{S}), \quad n^{a}=-i(\tau-\bar{\tau}) \bar{A}^{a}
$$

The covariant derivatives of $n^{a}$ are given by

$$
D_{\mu} n^{a} \equiv \partial_{\mu} n^{a}-(\tau-\bar{\tau})^{-1} \partial_{\mu} \tau\left(n^{a}-\bar{n}^{a}\right)
$$

and the scalar field $P$ in the Lagrangian (6) is the dual of the axion $\operatorname{Re} S .8$ Moreover we have chosen the conformal gauge for the $D=2$ metric

$$
g_{\mu \nu}^{(2)}=e^{\sigma} e^{2 \Phi_{\mathrm{het}}^{(2)}} \eta_{\mu \nu}
$$

Finally, the index $I$ now denotes all moduli except the dilaton and $\tilde{G}_{I \bar{J}}^{(4)}$ is the Kähler metric of the Kähler potential $\tilde{K}_{\text {het }}^{(4)}$ defined in eq. (2).

Defining the complexified Kähler modulus as

$$
\rho \equiv i \sqrt{h}+(\tau-\bar{\tau})^{-1} n^{a}\left(n^{a}-\bar{n}^{a}\right)+2 P
$$

one verifies that the Lagrangian (6) can be written in the form

$$
\mathcal{L}_{\text {het }}^{(2)}=e^{-2 \Phi_{\text {het }}^{(2)}}\left[-\frac{1}{2} \partial_{\mu} \partial^{\mu} \sigma-G_{\bar{\Lambda} \Sigma} \partial_{\mu} \bar{Z}^{\bar{\Lambda}} \partial^{\mu} Z^{\Sigma}\right],
$$

\footnotetext{
${ }^{7}$ The reason for taking the string-frame metric is that the variables which we get are exactly those we need in section 2.3 in order to establish the map to the type IIA variables in which the superpotential is naturally expressed. This will become clear in the following sections.

${ }^{8}$ More precisely we added the term $\partial_{\mu} P \epsilon^{\mu \nu} \partial_{\nu} \operatorname{Re} S$ to the reduced action and eliminated ReS by its equation of motion. One can also verify that $2 P$ is the $B_{23}$ component of the $D=4$ antisymmetric tensor in the compactified directions.
} 
where the $Z^{\Sigma}$ denote $Z^{\Sigma}=\left(\phi^{I}, \tau, \rho, n^{a}\right)$. The $Z^{\Sigma}$ are the proper Kähler coordinates in that in these coordinates $G_{\bar{\Lambda} \Sigma}=\bar{\partial}_{\bar{\Lambda}} \partial_{\Sigma} K_{\text {het }}^{(2)}$ with

$$
K_{\mathrm{het}}^{(2)}=\tilde{K}_{\mathrm{het}}^{(4)}(\phi, \bar{\phi})-\ln \left[\left(n^{a}-\bar{n}^{a}\right)^{2}-(\rho-\bar{\rho})(\tau-\bar{\tau})\right]
$$

The form (11) of the Lagrangian explicitly shows that both $\Phi_{\text {het }}^{(2)}$ and $\sigma$ are unphysical degrees of freedom [21] while $\rho$ and $\tau$ are propagating degrees of freedom. The four physical degrees of freedom in $\rho$ and $\tau$ are related to the four-dimensional graviton and the dilaton $S$.

In this parameterization the modular group of the torus $S L(2, \mathbb{Z}) \times S L(2, \mathbb{Z})$ acting on $\tau$ and $\rho$ as fractional linear transformation is manifest. The first $S L(2, \mathbb{Z})$ acts as

$$
\tau \rightarrow \frac{a \tau+b}{c \tau+d}, \quad \rho \rightarrow \rho-\frac{c n^{a} n^{a}}{c \tau+d}, \quad n^{a} \rightarrow \frac{n^{a}}{c \tau+d}, \quad\left(\begin{array}{ll}
a & b \\
c & d
\end{array}\right) \in S L(2, \mathbb{Z})
$$

and the action of the second $S L(2, \mathbb{Z})$ is obtained by exchanging $\tau$ and $\rho$.

$\tau$ and $\rho$ both reside in vector or twisted chiral multiplets since in the reduction procedure they come along with the Kaluza-Klein vectors.? From eq. (12) we learn that the moduli space factorizes into chiral multiplets $\phi^{I}$ and twisted chiral multiplets $\left(\tau, \rho, n^{a}\right)$ which span the coset space $S O(2,2+r) / S O(2) \times S O(2+r)$ where $r=\operatorname{rank}(G)$. In this case the two-dimensional $(2,2)$ supersymmetric $\sigma$-model is known to be Kähler [20], where the Kähler potential is a sum of two terms, one depending on the chiral multiplets and one depending on the twisted chiral multiplets. However, in more general situations the metric is not Kähler but nevertheless the Lagrangian can be expressed in terms of real functions similar to the Kähler potential. This situation occurs if one goes beyond the tree level approximation and includes string loop corrections. Some results about the general case are presented in appendix B. For completeness this appendix also contains a different derivation of the $D=2$ effective theory by compactifying the $D=3$ Lagrangian given in ref. [22] on a circle.

\subsection{Type IIA theory on Calabi-Yau fourfolds}

In this section we discuss the Kaluza-Klein reduction of type IIA on Calabi-Yau fourfolds. The starting point is the low energy effective action in $D=10$ in the string-frame

$$
\begin{aligned}
S_{I I A}^{(10)}= & \int d^{10} x \sqrt{-g^{(10)}} e^{-2 \Phi_{\mathrm{IIA}}^{(10)}}\left(\frac{1}{2} R^{(10)}+2 \partial_{M} \Phi_{\mathrm{IIA}}^{(10)} \partial^{M} \Phi_{\mathrm{IIA}}^{(10)}-\frac{1}{4}\left|H_{3}\right|^{2}\right) \\
& -\frac{1}{4} \int d^{10} x \sqrt{-g^{(10)}}\left(\left|F_{2}\right|^{2}+\left|\tilde{F}_{4}\right|^{2}\right)-\frac{1}{4} \int B_{2} \wedge F_{4} \wedge F_{4},
\end{aligned}
$$

where $\Phi_{\text {IIA }}^{(10)}$ is the ten-dimensional dilaton, $H_{3}=d B_{2}$ is the field strength of the antisymmetric tensor $B_{2}$ and $F_{2}=d C_{1}$ the field strength of the RR vector $C_{1} . F_{4}=d C_{3}$ is the field strength of the RR 3-form $C_{3}$ and we use the abbreviation $\tilde{F}_{4}=F_{4}-C_{1} \wedge H_{3}$. For further conventions on the notation see appendix A.

\footnotetext{
${ }^{9}$ The modulus $\rho$ is the dual of the chiral field $S$ and therefore twisted chiral. A further argument that $\tau$ is indeed part of a twisted chiral multiplet is given in appendix $B$.
} 
In eq. (14) only the leading terms of $S_{I I A}^{(10)}$ are displayed and higher derivative couplings are suppressed. In particular the term proportional to $\int B_{2} \wedge X_{8}, X_{8} \sim 4 \operatorname{tr} R^{4}-\left(\operatorname{tr} R^{2}\right)^{2}$ imposes a consistency condition on the compactification [3]. The absence of a $B_{2}$-tadpole requires

$$
-\int_{Y_{4}} X_{8}=\frac{\chi}{24}=n+\frac{1}{8 \pi^{2}} \int_{Y_{4}} F_{4} \wedge F_{4}
$$

where $n$ is the number of space-time filling strings. In this section we focus on the case $\chi=n=F_{4}=0$ and return to the case of non-trivial $F_{4}$ in section 3 .

The spectrum of the $D=2$ theory is determined by the deformations of the CalabiYau metric and the expansion of $C_{1}, B_{2}$ and $C_{3}$ in terms of the non-trivial forms of $Y_{4}$. The deformations of the metric comprise $h^{1,1}$ real Kähler deformations $M^{A}, A=1, \ldots, h^{1,1}$ and $h^{1,3}$ complex deformations $Z^{\alpha}, \alpha=1, \ldots, h^{1,3}$ of the complex structure. Since vectors contain no physical degree of freedom in $D=2$ and since there are no 1 -forms on $Y_{4}$ the 1-form $C_{1}$ does not contribute any massless mode in $D=2 . B_{2}$ leads to $h^{1,1}$ real scalar fields $a^{A}$ while $C_{3}$ contributes $h^{1,2}$ complex scalars $N^{I}, I=1, \ldots, h^{1,2}$. The $(1,1)$ moduli reside in twisted chiral multiplets 10 while all other scalars are members of chiral multiplets.

For simplicity let us discuss here only the case where the $(2,1)$-moduli are frozen to some fixed value and postpone the discussion of the general case to appendix C. Compactification of the action (14) results in

$$
\mathcal{L}_{I I A}^{(2)}=\sqrt{-g^{(2)}} e^{-2 \Phi_{\mathrm{IIA}}^{(2)}}\left(\frac{1}{2} R^{(2)}+2 \partial_{\mu} \Phi_{\mathrm{IIA}}^{(2)} \partial^{\mu} \Phi_{\mathrm{IIA}}^{(2)}-G_{A \bar{B}} \partial_{\mu} t^{A} \partial^{\mu} \bar{t}^{\bar{B}}-G_{\bar{\alpha} \beta} \partial_{\mu} \bar{Z}^{\bar{\alpha}} \partial^{\mu} Z^{\beta}\right)
$$

where the following definitions have been used

$$
\begin{aligned}
e^{-2 \Phi_{\mathrm{IIA}}^{(2)}} & \equiv e^{-2 \Phi_{\mathrm{IIA}}^{(10)}} \mathcal{V}, \\
t^{A} & \equiv \frac{1}{\sqrt{2}}\left(a^{A}+i M^{A}\right), \\
G_{A \bar{B}} & \equiv \frac{1}{2 \mathcal{V}} \int_{Y_{4}} e_{A} \wedge \star e_{B}=-\partial_{A} \bar{\partial}_{\bar{B}} \ln \mathcal{V}, \\
G_{\alpha \bar{\beta}} & \equiv-\frac{\int_{Y_{4}} \Phi_{\alpha} \wedge \bar{\Phi}_{\bar{\beta}}}{\int_{Y_{4}} \Omega \wedge \bar{\Omega}}=-\partial_{\alpha} \bar{\partial}_{\bar{\beta}} \ln \left[\int_{Y_{4}} \Omega \wedge \bar{\Omega}\right] .
\end{aligned}
$$

$e_{A}$ denotes a basis for the (1,1)-forms of $Y_{4}, \Phi_{\alpha}$ a basis for its (3,1)-forms and $\Omega$ is its unique $(4,0)$-form. $\mathcal{V}$ is the volume of the fourfold which can be expressed in terms of the Kähler form $J=M^{A} e_{A}$ as

$$
\mathcal{V}=\frac{1}{4 !} \int_{Y_{4}} J \wedge J \wedge J \wedge J=\frac{1}{4 \cdot 4 !} d_{A B C D}\left(t^{A}-\bar{t}^{A}\right)\left(t^{B}-\bar{t}^{B}\right)\left(t^{C}-\bar{t}^{C}\right)\left(t^{D}-\bar{t}^{D}\right)
$$

where $d_{A B C D}$ are the classical intersection numbers $d_{A B C D}=\int_{Y_{4}} e_{A} \wedge e_{B} \wedge e_{C} \wedge e_{D}$.

\footnotetext{
${ }^{10}$ Strictly speaking these multiplets are vector multiplets containing in addition the vectors arising from expanding the 3 -form in terms of the $(1,1)$-forms of $Y_{4}$. But as noted in the last section, a vector multiplet is related to a twisted chiral multiplet, differing only in the auxiliary field content.
} 
As in the heterotic case the moduli space factorizes into chiral and twisted chiral multiplets, i.e. it is Kähler although both kinds of multiplets occur. The Kähler potential can be read off from (17) and is given by

$$
K_{I I A}^{(2)}=-\ln \left(\int_{Y_{4}} \Omega \wedge \bar{\Omega}\right)-\ln \mathcal{V}
$$

If $(2,1)$-moduli arising from $C_{3}$ are taken into account the metric ceases to be Kähler but still can be expressed in terms of a real function. This situation is discussed in appendix Q.円

Mirror symmetry is believed to be a property of all Calabi-Yau manifolds and for fourfolds it exchanges $(1,1)$ - and $(1,3)$-forms [23, 1, 24]. As it stands the Kähler potential (19) does not share this symmetry due to the fact that the derivation used the supergravity approximation which is only valid if the size of the manifold is large compared to the string length (i.e. in the large radius approximation). For 'small' fourfolds worldsheet instantons correct the Kähler potential of the $(1,1)$-moduli and are believed to render (19) mirror symmetric.

\subsection{Heterotic - type IIA duality in $\mathrm{D}=2$}

In order to establish the duality relationship between the heterotic and type IIA variables we specify the compactification manifolds. The Calabi-Yau fourfold is taken to be a $K 3$ fibration over a large Hirzebruch surface $\mathbb{F}_{n}$ with $n$ even while the Calabi-Yau threefold on the heterotic side is an elliptic fibration over the same base. In the large base limit the type IIA Kähler potential simplifies as [22]

$$
K_{I I A}^{(2)}=-\ln \mathcal{V} \rightarrow-\ln \left[\left(t^{U}-\bar{t}^{U}\right)\left(t^{V}-\bar{t}^{V}\right) \eta_{\hat{\imath} \hat{\jmath}}\left(t^{\hat{\imath}}-\bar{t}^{\hat{\imath}}\right)\left(t^{\hat{\jmath}}-\bar{t}^{\hat{\jmath}}\right)\right]
$$

where $t^{U}$ and $t^{V}$ are the moduli of the base while the $t^{\hat{\imath}}$ denote the moduli of the $K 3$-fibre except those from reducible bad fibres. $\eta$ is the intersection matrix of $K 3$

$$
\eta_{\hat{\imath} \hat{\jmath}}=\left(\begin{array}{ccc}
0 & 1 / 2 & \\
1 / 2 & 0 & \\
& & -\mathbf{I}
\end{array}\right) \text {. }
$$

On the heterotic side the Kähler potential (12) becomes in the large base limit

$$
K_{\text {het }}^{(2)}=-\ln \left[(U-\bar{U})(V-\bar{V})\left((\tau-\bar{\tau})(\rho-\bar{\rho})-\left(n^{a}-\bar{n}^{a}\right)^{2}\right)\right],
$$

where $U$ and $V$ are the two base moduli. Comparing (20) and (22) it is tempting to equate the two expressions. However, this would map the twisted chiral superfields $t^{U}$ and $t^{V}$ to chiral superfields $U$ and $V$. Hence one has to first perform an additional

\footnotetext{
${ }^{11}$ The same appendix also presents a different derivation of the $D=2$ effective theory where an $S^{1}$ reduction of the three-dimensional effective theory of ref. [22 obtained as compactification of $D=11$ supergravity on Calabi-Yau fourfolds $Y_{4}$ is performed. In this case all scalar fields appear naturally as members of chiral multiplets and as a consequence the metric in these coordinates is always Kähler. The Kähler potential of the $D=2$ effective theory coincides with the Kähler potential of the $D=3$ effective theory.
} 
duality transformation on the base moduli $U, V$ so that all heterotic variables are twisted chiral like their type IIA counterparts. This is possible in the large base limit by defining $c_{U}^{\nu}=-\epsilon^{\mu \nu} \partial_{\mu} \operatorname{Re} U$ and $c_{V}^{\nu}=-\epsilon^{\mu \nu} \partial_{\mu} \operatorname{Re} V$ and add the Lagrange multipliers $P_{U}, P_{V}$ via $-c_{U}^{\mu} \partial_{\mu} P_{U}-c_{V}^{\mu} \partial_{\mu} P_{V}$ to the action. Using the equations of motion to eliminate $c_{U}^{\mu}$ and $c_{V}^{\mu}$, defining the coordinates

$$
u \equiv 2 P_{U}+i e^{-2 \Phi_{\text {het }}^{(2)}(\operatorname{Im} U)^{-1}}, \quad v \equiv 2 P_{V}+i e^{-2 \Phi_{\text {het }}^{(2)}(\operatorname{Im} V)^{-1}}
$$

and redefining the $D=2$ metric

$$
g_{\mu \nu}^{(2)}=e^{\sigma} e^{4 \Phi_{\mathrm{het}}^{(2)}} \operatorname{Im} U \operatorname{Im} V \eta_{\mu \nu}
$$

one derives the following form of the Kähler potential

$$
\hat{K}_{\text {het }}^{(2)}=-\ln \left[(u-\bar{u})(v-\bar{v})\left((\tau-\bar{\tau})(\rho-\bar{\rho})-\left(n^{a}-\bar{n}^{a}\right)^{2}\right)\right] .
$$

In this form all the coordinates belong to twisted chiral multiplets. Hence the duality map relates[

$$
\begin{aligned}
\left\{t^{\hat{\imath}}\right\} & \leftrightarrow\left\{\tau, \rho, n^{a}\right\}, \\
\left\{t^{U}, t^{V}\right\} & \leftrightarrow\{u, v\} .
\end{aligned}
$$

As we discussed in section 2.1 the heterotic theory has an $S L(2, \mathbb{Z}) \times S L(2, \mathbb{Z})$ symmetry (13) which is just the modular symmetry of the torus. From the map (26) one learns that in the type IIA theory this symmetry has to be a property of $K 3$-fibred fourfolds in the large base limit. This is precisely the same situation one encounters in the four-dimensional duality relating type IIA compactified on $K 3$-fibred threefolds to heterotic vacua compactified on $K 3 \times T^{2}$ [25].

A similar analysis was performed in $D=3$ in ref. [22]. As mentioned in the previous sections one can derive the two-dimensional effective actions via the detour over $D=3$. In this case the scalars are members of chiral multiplets only and therefore parameterize a Kähler manifold with the same Kähler potential as in $D=3$. Thus the map derived in [22] in the corresponding coordinates continues to hold in $D=2$. However, for the purpose of this paper the map (26) turns out to be more useful. For further details we refer the reader to appendix $\mathrm{C}$.

\section{$3 \quad$ Type IIA string with background fluxes}

\subsection{The superpotential in IIA}

In this section we consider compactifications of type IIA string theory on Calabi-Yau fourfolds in a background where non-trivial RR-fluxes have been turned on and simultaneously all $(1,2)$ moduli have been frozen. In refs. [7, 9] it was shown that the low

\footnotetext{
${ }^{12}$ The fact that it is really $u$ and $v$ which should be mapped to $t^{U}$ and $t^{V}$ can heuristically be understood from the duality in $D=6$ fibred over a four-dimensional base manifold $B$. It yields $\mathcal{V}_{B}^{\text {IIA }}=$ $e^{-4 \Phi_{\text {het }}^{(6)}} \mathcal{V}_{B}^{\text {het }}=e^{-2 \Phi_{\text {het }}^{(6)}} e^{-2 \Phi_{\text {het }}^{(2)}}$ and similarly $\mathcal{V}_{B}^{\text {het }}=e^{-2 \Phi_{\text {IIA }}^{(6)}} e^{-2 \Phi_{\text {IIA }}^{(2)}}=e^{2 \Phi_{\text {het }}^{(6)}} e^{-2 \Phi_{\text {IIA }}^{(2)}}$, where all volumes are those of the base measured in the corresponding string-frame metric. Using (C.27) one verifies immediately $\mathcal{V}_{B}^{\text {IIA }}=\left(\mathcal{V}_{B}^{\text {het }} e^{4 \Phi_{\text {het }}^{(2)}}\right)^{-1}$.
} 
energy effective theory can be described by a $(2,2)$-supersymmetric Lagrangian with a non-trivial potential which depends on the background fluxes. The potential $V$ is derived from two superpotentials $W$ and $\tilde{W}$ [26]

$$
V \sim e^{K_{I A}^{(2)}}\left(G^{-1 A \bar{B}} D_{A} \tilde{W} D_{\bar{B}} \overline{\tilde{W}}+G^{-1 \alpha \bar{\beta}} D_{\alpha} W D_{\bar{\beta}} \bar{W}-|W|^{2}-|\tilde{W}|^{2}\right)
$$

where $W$ depends on the chiral complex structure scalars $Z^{\alpha}$ and $\tilde{W}$ on the twisted chiral Kähler scalars $t^{A}$. The Kähler covariant derivatives are defined as

$$
D_{A} \tilde{W}=\partial_{A} \tilde{W}+\tilde{W} \partial_{A} K_{I I A}^{(2)}, \quad D_{\alpha} W=\partial_{\alpha} W+W \partial_{\alpha} K_{I I A}^{(2)}
$$

Since D-branes are the magnetic sources of RR-fluxes the generation of the superpotentials can also be understood from wrapping the D-branes in the IIA theory on supersymmetric cycles. More specifically, wrapping a D4-brane on a four-dimensional special Lagrangian cycle generates the superpotential $[7,9]]^{3}$

$$
W=\frac{1}{2 \pi} \int_{Y_{4}} \Omega \wedge F_{4}
$$

where $F_{4}$ denotes the RR 4-form flux.

A second superpotential is generated by wrapping $\mathrm{D} p$-branes, $p=0,2,4,6,8$, on holomorphic cycles $C^{(p)} \in H_{p}\left(Y_{4}, \mathbb{Z}\right)$ with the same real dimension $p$ [7, 9]

$$
\tilde{W}_{\mathrm{cl}}=\frac{1}{2 \pi} \int_{Y_{4}}\left(t \wedge t \wedge t \wedge t F_{0}+t \wedge t \wedge t \wedge F_{2}+t \wedge t \wedge F_{4}+t \wedge F_{6}+F_{8}\right)
$$

where $t=t^{A} e_{A}$ and $\frac{F_{p}}{2 \pi} \in H^{p}\left(Y_{4}, \mathbb{Z}\right)$ is the RR $p$-form flux which is Poincare dual to the $(8-p)$-cycle $C^{(8-p)} \in H_{8-p}\left(Y_{4}, \mathbb{Z}\right)$ (for $p=4$ see the last footnote). ${ }^{\mathbb{4}}$

The effective theory has $(2,2)$ supersymmetry in a Minkowskian background if

$$
\left.D_{A} \tilde{W}\right|_{\min }=\left.D_{\alpha} W\right|_{\min }=\left.\tilde{W}\right|_{\min }=\left.W\right|_{\min }=0
$$

holds. Depending on the background fluxes this puts a severe constraint on the moduli space and for some fluxes no supersymmetric vacuum exists at all. For example, for the $W$ of eq. (29) the supersymmetry condition (31) implies [29]

$$
F_{4}^{(0,4)}=0=F_{4}^{(1,3)}
$$

where the last equation arises from the fact that $\partial_{\alpha} \Omega$ takes values in $H^{4,0}$ and in $H^{3,1}$. Since the Hodge decomposition of $H^{4}$ depends on the complex structure, eq. (32) is a strong constraint on the moduli space of the complex structure. It leaves only the

\footnotetext{
${ }^{13}$ As argued in [7] the change of the superpotential when crossing the brane in the two-dimensional space-time is equal to the volume of the four-cycle $C^{(4)} \in H_{4}\left(Y_{4}, \mathbb{Z}\right)$ wrapped by the $\mathrm{D} 4$-brane: $\Delta W=$ $\int_{C^{(4)}} \Omega=\frac{1}{2 \pi} \int_{Y_{4}} \Omega \wedge \Delta F_{4}$. The change of the 4-form flux $\frac{\Delta F_{4}}{2 \pi} \in H^{4}\left(Y_{4}, \mathbb{Z}\right)$ is Poincaré dual to the four-cycle $C^{(4)}$. Note that $\frac{F_{4}}{2 \pi}$ itself does in general not take values in $H^{4}\left(Y_{4}, \mathbb{Z}\right)$ but $\frac{F_{4}}{2 \pi}-\frac{p_{1}}{4}$, where $p_{1}$ is the first Pontryagin class [27].

${ }^{14}$ It should be possible to derive $W, \tilde{W}$ also from a KK-reduction as outlined in the previous section where appropriate fluxes have been turned on [10, 28].
} 
subspace of complex structure deformations which respect (32) as the physical moduli space.

The superpotential $\tilde{W}$ receives quantum corrections on the worldsheet while $W$ is exact. Mirror symmetry demands that once all quantum corrections are properly taken into account the two superpotentials should obey [30, 5, 9]

$$
\tilde{W}\left(Y_{4}\right)=\tilde{W}_{\mathrm{cl}}\left(Y_{4}\right)+\text { quantum corrections }=W\left(\tilde{Y}_{4}\right)
$$

where $\tilde{Y}_{4}$ is the mirror fourfold of $Y_{4}$. The quantum corrections can be derived by computing $W$ on the mirror manifold and performing the mirror map. This is of interest since in the dual heterotic vacuum the $(1,1)$-scalars correspond to the heterotic variables $\left\{\tau, \rho, n^{a}\right\}$ (c.f. (26)) which are related to the four-dimensional heterotic dilaton. Thus the quantum corrected $\tilde{W}\left(Y_{4}\right)$ possibly encodes non-trivial information about space-time quantum corrections of four-dimensional heterotic vacua. As we will see the superpotential evaluated in the large base limit of $K 3$-fibred fourfolds which are simultaneously $Y_{3}$-fibred is determined by the prepotential $\mathcal{F}$ of $Y_{3}$ if the background values for $F_{p}$ are suitably chosen.

\subsection{The superpotential for threefold-fibred fourfolds}

Let us first introduce the notion of the vertical primary subspace 23, 4]. This is the subspace of $\oplus_{k=0}^{d} H^{(k, k)}\left(Y_{d}\right)$ obtained by taking all wedge products of the $(1,1)$-forms. The horizontal primary subspace of $\oplus_{k=0}^{d} H^{(d-k, k)}\left(Y_{d}\right)$ can be obtained from the vertical primary subspace of the mirror manifold via mirror symmetry [23, 4, 24]. T. Let us consider the class of fourfolds which are $Y_{3}$-fibred over a base $\mathbb{P}^{1}[4$. Assuming the absence of reducible bad fibres in this $Y_{3}$-fibration the vertical primary subspace can be obtained by taking the wedge product of the elements of the vertical primary subspace of $Y_{3}$ with the zero- or $(1,1)$-forms on the $\mathbb{P}^{1}$ base. This leads to the following Hodge numbers of the vertical primary subspace of $Y_{4}$

$$
\begin{aligned}
& h^{(0,0)}\left(Y_{4}\right)=1=h^{(4,4)}\left(Y_{4}\right), \\
& h^{(1,1)}\left(Y_{4}\right)=h^{(1,1)}\left(Y_{3}\right)+1=h^{(3,3)}\left(Y_{4}\right), \\
& h_{V}^{(2,2)}\left(Y_{4}\right)=2 h^{(1,1)}\left(Y_{3}\right) .
\end{aligned}
$$

Except for $h_{V}^{(2,2)}$ the Hodge numbers of the vertical primary subspace coincide with those of the full vertical cohomology. In the following we also use the formulation of the vertical primary subspace in terms of the dual homology. In the homology the vertical primary subspace is obtained by joining the even-dimensional cycles of $Y_{3}$ with the zeroor two-cycles of the base $\mathbb{P}^{1}$.

Mirror symmetry implies the following relations for the Hodge numbers of the horizontal primary subspace of $\tilde{Y}_{4}$

$$
\begin{array}{ll}
h^{(0,4)}\left(\tilde{Y}_{4}\right)=h^{(4,4)}\left(Y_{4}\right), & h^{(4,0)}\left(\tilde{Y}_{4}\right)=h^{(0,0)}\left(Y_{4}\right), \\
h^{(3,1)}\left(\tilde{Y}_{4}\right)=h^{(1,1)}\left(Y_{4}\right), & h_{H}^{(2,2)}\left(\tilde{Y}_{4}\right)=h_{V}^{(2,2)}\left(Y_{4}\right) .
\end{array}
$$

\footnotetext{
${ }^{15}$ The horizontal primary subspace is generated by successive derivatives of the holomorphic $(d, 0)$-form $\Omega$ 31, 23.
} 
The members of the vertical respectively the horizontal primary cohomology are observables in the A- respectively B-model. The A- and the B-model are two topological sigma-models with the Calabi-Yau manifold $Y_{4}$ as a target space, which are obtained by twisting the worldsheet sigma-model in two different ways [32]. The observables and correlation functions of the A-model on $Y_{4}$ are related via mirror symmetry to those of the B-model on $\tilde{Y}_{4}$ and vice versa.

\subsubsection{Computation of $W\left(\tilde{Y}_{4}\right)$ and $\tilde{W}\left(Y_{4}\right)$}

Our goal is to compute $\tilde{W}\left(Y_{4}\right)$ in the large base limit using mirror symmetry and eqs. (33) and (29). This is possible if we choose the background fluxes $F_{p}$ on $Y_{4}$ to lie in the vertical primary subspace. These fluxes are mapped to elements of the horizontal primary subspace on the mirror manifold $\tilde{Y}_{4}$. The dimension $h_{H}^{4}\left(\tilde{Y}_{4}\right)$ of the horizontal primary subspace is given by

$$
h_{H}^{4}=2 h^{(4,0)}\left(\tilde{Y}_{4}\right)+2 h^{(3,1)}\left(\tilde{Y}_{4}\right)+h_{H}^{(2,2)}\left(\tilde{Y}_{4}\right)=4\left(h^{(1,1)}\left(Y_{3}\right)+1\right)
$$

Let us denote a basis for the horizontal primary homology of $\tilde{Y}_{4}$ by $\left(A^{I}, \tilde{A}_{I}, B^{I}, \tilde{B}_{I}\right)$, where $I=0, \ldots, h^{(1,1)}\left(Y_{3}\right)$. The $\left(A^{I}, \tilde{A}_{I}\right)$ are those homology cycles that correspond via mirror symmetry to the elements of the vertical primary subspace of $Y_{4}$ which are obtained by joining the even-dimensional cycles of the threefold-fibre with the zero-cycle of the base. Analogously, $\left(B^{I}, \tilde{B}_{I}\right)$ are the cycles which are related to the elements of the vertical primary subspace which are obtained by joining the even-dimensional cycles of the threefold with the two-cycle of the base [4]. As noted in [4] in the large base limit at leading order the cycles $\left(A^{I}, \tilde{A}_{I}\right)$ all have vanishing intersections with each other and the same is true for the $\left(B^{I}, \tilde{B}_{I}\right)$ cycles. The only non-vanishing intersections are between $A$-cycles and $B$-cycles and the intersection form is given by that of the $Y_{3}$-fibre. For a certain choice of cycles and in terms of the Poincaré dual forms $\left(a^{I}, \tilde{a}_{I}, b^{I}, \tilde{b}_{I}\right)$ this amounts to

$$
\int_{\tilde{Y}_{4}} a^{I} \wedge \tilde{b}_{J}=\delta_{J}^{I}=-\int_{\tilde{Y}_{4}} \tilde{a}_{J} \wedge b^{I}
$$

with all other intersection pairings vanishing.

In order to evaluate the superpotential $W\left(\tilde{Y}_{4}\right)$ using eq. 29) we expand the 4-form flux on $\tilde{Y}_{4}$ in this basis

$$
\frac{\tilde{F}_{4}}{2 \pi}=\mu_{I} a^{I}-\tilde{\mu}^{I} \tilde{a}_{I}+\nu_{I} b^{I}-\tilde{\nu}^{I} \tilde{b}_{I}, \quad\left(\mu_{I}, \tilde{\mu}^{I}, \nu_{I}, \tilde{\nu}^{I}\right) \in \mathbb{Z} .
$$

(We denote it by $\tilde{F}_{4}$ in order to distinguish it from the 4-form flux on $Y_{4}$.) The fluxes are not all independent but have to obey the consistency condition (15). This implies

$$
\frac{1}{24} \chi\left(\tilde{Y}_{4}\right)=n+\left(\nu_{I} \tilde{\mu}^{I}-\mu_{I} \tilde{\nu}^{I}\right)
$$

Inserting (38) into (29) we arrive at

$$
W=\mu_{I} \int_{A^{I}} \Omega-\tilde{\mu}^{I} \int_{\tilde{A}_{I}} \Omega+\nu_{I} \int_{B^{I}} \Omega-\tilde{\nu}^{I} \int_{\tilde{B}_{I}} \Omega .
$$


In order to evaluate the period integrals $\int \Omega$ on $\tilde{Y}_{4}$ one has to note that they are mapped via mirror symmetry to the periods on $Y_{4}$, which give to leading order in the large volume limit and in special coordinates the classical volumes of the corresponding cycles. These leading terms in general get quantum corrections. In the large base limit the corrections from the base are suppressed and the quantum corrections only arise from the threefold fibre. Thus the periods on the fourfold are given by those of the threefold, multiplied by the classical volume $t^{V}$ of the base for those cycles which contain the base [4]. More specifically one has

$$
\begin{aligned}
\int_{A_{I}} \Omega & =\left(1, t^{i}\right)+\mathcal{O}\left(e^{i t^{V}}\right), \quad \int_{B_{I}} \Omega=t^{V}\left(1, t^{i}\right)+\mathcal{O}\left(\left(t^{V}\right)^{0}\right)+\mathcal{O}\left(e^{i t^{V}}\right), \\
\int_{\tilde{A}_{I}} \Omega & =\left(\mathcal{F}_{i}, \mathcal{F}_{0}\right)+\mathcal{O}\left(e^{i t^{V}}\right),
\end{aligned}
$$

where $i=1, \ldots, h^{(1,1)}\left(Y_{3}\right)$. The vector $\Pi=\left(1, t^{i}, \mathcal{F}_{i}, \mathcal{F}_{0}\right)$ corresponds to the periods of the threefold with $\mathcal{F}_{i}=\partial_{t^{i}} \mathcal{F}$ and $\mathcal{F}_{0}=2 \mathcal{F}-t^{i} \mathcal{F}_{i}$. The $N=2$ prepotential is given by 33

$$
\mathcal{F}=\mathcal{F}_{\text {pol }}-\frac{1}{(2 \pi)^{3}} \sum_{\left\{d_{i}\right\}} n_{\left\{d_{i}\right\}} L i_{3}\left(e^{2 \pi i \sum t^{i} d_{i}}\right)
$$

where

$$
\mathcal{F}_{\text {pol }}=\frac{1}{6} d_{i j k} t^{i} t^{j} t^{k}+b_{i} t^{i}+\frac{1}{2} c, \quad L i_{3}(x) \equiv \sum_{j=1}^{\infty} \frac{x^{j}}{j^{3}}
$$

The $d_{i j k}$ are the classical intersection numbers of the threefold and the coefficients $b_{i}, c$ are given in [33]. The $d_{i}$ are the instanton numbers of the $i$-th $(1,1)$-form, $d_{i}=\int_{\mathcal{C}} e_{i}$, while $n_{\left\{d_{i}\right\}}$ is the number of isolated holomorphic curves $\mathcal{C}$ of multi-degree $\left(d_{1}, \ldots, d_{h^{(1,1)}}\right)$ in the threefold fibre and the sum over $j$ takes into account multiple coverings.

Inserting (41) into (40) and assuming that the $\mu_{I}$ and $\tilde{\mu}^{I}$ are large so that the $\mathcal{O}\left(\left(t^{V}\right)^{0}\right)$ term can be neglected we arrive at

$$
W\left(\tilde{Y}_{4}\right)=\mu_{0}+\mu_{i} t^{i}-\tilde{\mu}^{i} \mathcal{F}_{i}-\tilde{\mu}^{0} \mathcal{F}_{0}+\nu_{0} t^{V}+\nu_{i} t^{i} t^{V}-\tilde{\nu}^{i} \mathcal{F}_{i} t^{V}-\tilde{\nu}^{0} \mathcal{F}_{0} t^{V}
$$

For future reference let us recall that the same period integrals (41) have been used in (41) to derive the Kähler potential in the large base limit to be

$$
K_{I I A}^{(2)}=-\ln \left[\int_{\tilde{Y}_{4}} \Omega \wedge \bar{\Omega}\right]=-\ln \left[\left(t^{V}-\bar{t}^{V}\right)\left(2(\mathcal{F}-\overline{\mathcal{F}})-\left(t^{i}-\bar{t}^{i}\right)\left(\mathcal{F}_{i}+\overline{\mathcal{F}}_{i}\right)\right)\right]
$$

$W$ can be written in a more suggestive way by expressing it not in terms of special coordinates $t^{i}$ but rather in terms of the homogeneous coordinates $X^{I}$ [33]. These coordinates are commonly used in $N=2$ supergravity and are holomorphic functions of the special coordinates $X^{I}\left(t^{i}\right)$. Furthermore, one has a prepotential $F(X)$ which is a homogeneous function of the $X^{I}$ of degree two. The special coordinates are just the particular

\footnotetext{
${ }^{16}$ We thank P. Mayr for a clarifying discussion concerning the period integrals.
} 
coordinate choice $t^{0}=X^{0} / X^{0}=1, t^{i}=X^{i} / X^{0}, \mathcal{F}\left(t^{i}\right)=\left(X^{0}\right)^{-2} F(X)$. In homogeneous coordinates the periods of the threefold are

$$
\Pi=\left(X^{I}, F_{I}\right)=X^{0}\left(1, t^{i}, \mathcal{F}_{i}, \mathcal{F}_{0}\right)
$$

while the Kähler potential reads

$$
K_{I I A}^{(2)}=-\ln \left[\left(t^{V}-\bar{t}^{V}\right)\left(\bar{X}^{I} F_{I}-X^{I} \bar{F}_{I}\right)\right] .
$$

This coincides with the Kähler potential given in (45) up to a Kähler transformation that amounts to a different normalization of the (4,0)-form $\Omega$. Inserting (46) into (44) finally yields

$$
W\left(\tilde{Y}_{4}\right)=\alpha_{I} X^{I}-\beta^{I} F_{I}
$$

where we abbreviated $\alpha_{I}=\mu_{I}+t^{V} \nu_{I}, \beta^{I}=\tilde{\mu}^{I}+t^{V} \tilde{\nu}^{I}$ and discarded an $X^{0}$-factor by the same Kähler transformation.

Curiously the superpotential (48) coincides with the superpotential derived in ref. [11]. It arises in type IIB compactifications on a Calabi-Yau threefold with non-vanishing RRand NS 3-form fluxes studied in refs. [13, 11, 14]. In this case the type IIB dilaton plays the role of $t^{V}$ in (48). It also is very closely related to the BPS-mass formula studied in refs. [15, 16] and the entropy formula of $N=2$ black holes [17]. This fortunate coincidence saves us from a detailed analysis of the supersymmetric minima of (48) and we can simply refer the reader to refs. [13, 11, 14, 34, 16]. One finds that for generic fluxes no supersymmetric vacuum exists. However, if the $\alpha_{I}, \beta^{I}$ are appropriately chosen supersymmetric ground states can exists. This can happen if the fluxes are aligned with cycles of the threefold which can degenerate at specific points in the moduli space [12, 13, 11, 14, 34]. These points (or subspaces) then correspond to supersymmetric ground states. They also coincide with the supersymmetric attractor points studied in refs. [17]. Note that in ref. [11] the consistency of the compactification required $\nu_{I} \tilde{\mu}^{I}-$ $\mu_{I} \tilde{\nu}^{I}=0$ while in our case this is replaced with the generalized condition given in eq. (39).

Before we evaluate $\tilde{W}\left(Y_{4}\right)$ let us briefly discuss the symmetry properties of $W\left(\tilde{Y}_{4}\right)$ as obtained in eq. (48). In homogeneous coordinates the period vector $\Pi$ transforms as a symplectic vector according to

$$
\left(\begin{array}{c}
F_{I} \\
X^{I}
\end{array}\right) \rightarrow\left(\begin{array}{cc}
A & B \\
C & D
\end{array}\right)\left(\begin{array}{c}
F_{I} \\
X^{I}
\end{array}\right), \quad\left(\begin{array}{cc}
A & B \\
C & D
\end{array}\right) \in \operatorname{Sp}\left(2 h^{(1,1)}+2, \mathbb{Z}\right)
$$

This transformation leaves the symplectic product $\left(\bar{X}^{I} F_{I}-X^{I} \bar{F}_{I}\right)$ invariant and via eq. (47) also the Kähler potential (in homogeneous coordinates). The fact that $\left(F_{I}, X^{I}\right)$ transform as a symplectic vector implies that also $\left(\tilde{a}_{I}, a^{I}\right)$ and $\left(\tilde{b}_{I}, b^{I}\right)$ transform according to (49). Indeed one verifies that the intersection matrix given in (37) is left invariant if $\left(\tilde{a}_{I}, a^{I}\right)$ as well as $\left(\tilde{b}_{I}, b^{I}\right)$ transform as symplectic vectors. Since $\tilde{F}_{4}$ has to be symplectically invariant one infers from $(38)$ that in turn the fluxes $\left(\mu_{I}, \tilde{\mu}^{I}\right)$ and $\left(\nu_{I}, \tilde{\nu}^{I}\right)$ have to transform as symplectic vectors, i.e. according to (49). Since $t^{V}$ is invariant we conclude that $\left(\alpha_{I}, \beta^{I}\right)$ form a symplectic vector and $W$ of $(48)$ is invariant.

Having derived the expression (44) for $W\left(\tilde{Y}_{4}\right)$ we can use eq. (33) to determine the quantum corrections of $\tilde{W}\left(Y_{4}\right)$. By matching the classical terms of $\tilde{W}\left(Y_{4}\right)$ as given in eq. 
(30) with the classical part of (44) using (42), (43) one is led to the identification

$$
\begin{aligned}
& -\tilde{\nu}^{0} \mathcal{F}_{0} t^{V}=\frac{1}{2 \pi} \int_{Y_{4}} t \wedge t \wedge t \wedge t F_{0}+\text { quantum corrections } \\
& -\tilde{\mu}^{0} \mathcal{F}_{0}-\tilde{\nu}^{i} \mathcal{F}_{i} t^{V}=\frac{1}{2 \pi} \int_{Y_{4}} t \wedge t \wedge t \wedge F_{2}+\text { q.c. }, \\
& \nu_{i} t^{i} t^{V}-\tilde{\mu}^{i} \mathcal{F}_{i}=\frac{1}{2 \pi} \int_{Y_{4}} t \wedge t \wedge F_{4}+\text { q.c. }, \\
& \nu_{0} t^{V}+\mu_{i} t^{i}=\frac{1}{2 \pi} \int_{Y_{4}} t \wedge F_{6} \\
& \mu_{0}=\frac{1}{2 \pi} \int_{Y_{4}} F_{8}=\text { const. . }
\end{aligned}
$$

The right hand sides of (53), (54) are two- and one-point functions in the topological A-model and therefore do not receive instanton corrections [23, 35]. From eq. (52) using (42), (43) we learn that the term including $\mathcal{F}_{i}$ has a polynomial piece and instanton corrections while the second term $\nu_{i} t^{i} t^{V}$ is purely classical. This can also be understood by considering the corresponding correlation functions in the A-model. Let us now go through this computation in more detail.

\subsubsection{The superpotential $\tilde{W}$ generated by 4 -form flux}

We denote the observables of the A-model by $\mathcal{O}_{M}^{(k)} \in H^{(k, k)}\left(Y_{d}\right)$, where the index takes the values $M=1, \ldots, h_{V}^{(k, k)}\left(Y_{d}\right)$. The two-point functions

$$
\eta_{M N}^{(k)}=\left\langle\mathcal{O}_{M}^{(k)} \mathcal{O}_{N}^{(d-k)}\right\rangle=\int_{Y_{d}} \mathcal{O}_{M}^{(k)} \wedge \mathcal{O}_{N}^{(d-k)}
$$

get no instanton contributions and define a flat metric on the vertical primary cohomology [35]. 17 On the other hand the three-point functions

$$
Y_{K L M}^{(k)}=\left\langle\mathcal{O}_{K}^{(1)} \mathcal{O}_{L}^{(k)} \mathcal{O}_{M}^{(d-k-1)}\right\rangle
$$

do receive instanton corrections. Because of their factorization properties all other amplitudes can be expressed in terms of the two- and three-point functions $\eta_{M N}^{(k)}, Y_{K L M}^{(k)}$ [23.

Choosing the 4-form flux as

$$
\frac{F_{4}}{2 \pi}=\sum_{N=1}^{h_{V}^{2,2}} \lambda^{N} \mathcal{O}_{N}^{(2)}
$$

ref. [5] proposed the following formula

$$
\partial_{t^{A}} \partial_{t^{B}} \tilde{W}\left(Y_{4}\right)=\sum_{N} \lambda^{N}\left\langle\mathcal{O}_{A}^{(1)} \mathcal{O}_{B}^{(1)} \mathcal{O}_{N}^{(2)}\right\rangle
$$

\footnotetext{
${ }^{17}$ Note that this metric is not the Zamolodchikov metric $G_{A \bar{B}}=\partial_{A} \partial_{\bar{B}} K$ which does receive quantum corrections as can be seen from eq. (45).
} 
In the following we evaluate this three-point function for a threefold-fibred fourfold in the large base limit and show that (58) is consistent with (52).

Let us first consider the part of $F_{4}$ which has one component in the base

$$
\frac{F_{4}}{2 \pi}=\sum_{N=1}^{h^{1,1}\left(Y_{3}\right)} \lambda^{N} \mathcal{O}_{N}^{(2)}=\sum_{i=1}^{h^{(1,1)}\left(Y_{3}\right)} \lambda^{V i} e_{V} \wedge e_{i}
$$

where $\lambda^{V i}$ is large. In the large base limit, there are no instanton corrections from the base so that according to the classical intersection numbers $e_{V}$ occurs at most once in any correlation function. The divisor which is dual to $e_{V}$ is the threefold-fibre and projects the amplitude of the fourfold to the threefold. In particular, for the three-point function with the 4-form flux as in (59), the three-point function on the fourfold is projected to the three-point function on the threefold [4]

$$
\partial_{t^{i}} \partial_{t^{j}} \tilde{W}\left(Y_{4}\right)=\sum_{N} \lambda^{N}\left\langle\mathcal{O}_{i}^{(1)} \mathcal{O}_{j}^{(1)} \mathcal{O}_{N}^{(2)}\right\rangle_{Y_{4}}=\sum_{k} \lambda^{V k}\left\langle\mathcal{O}_{i}^{(1)} \mathcal{O}_{j}^{(1)} \mathcal{O}_{k}^{(1)}\right\rangle_{Y_{3}}=\sum_{k} \lambda^{V k} Y_{i j k}
$$

where

$$
\mathcal{O}_{N}^{(2)}=e_{V} \wedge e_{k}, \quad \mathcal{O}_{k}^{(1)}=e_{k}
$$

In special coordinates the three-point function $Y_{i j k}$ on $Y_{3}$ is the third derivative of a holomorphic prepotential [33]

$$
Y_{i j k}=\mathcal{F}_{i j k}, \quad \mathcal{F}_{i j k}=\partial_{t^{i}} \partial_{t^{j}} \partial_{t^{k}} \mathcal{F} .
$$

Inserting (62) in eq. (60) we learn that $\partial_{t^{i}} \partial_{t^{j}} \tilde{W}\left(Y_{4}\right)$ can be integrated and indeed coincides with the instanton corrected part of the expression (52).

In order to get the full superpotential, we still have to consider the part with the 4-form flux restricted to the threefold fibre, i.e.

$$
\frac{F_{4}}{2 \pi}=\sum_{i=1}^{h^{1,1}\left(Y_{3}\right)} \lambda^{i} \mathcal{O}_{i}^{(2)}
$$

We have to distinguish two cases. First we consider the three-point function which contains one observable $\mathcal{O}^{(1)}$ corresponding to the base. In this case the three-point function of the fourfold is projected to the two-point function of the threefold fibre:

$$
\partial_{t^{V}} \partial_{t^{j}} \tilde{W}\left(Y_{4}\right)=\lambda^{i}\left\langle\mathcal{O}_{V}^{(1)} \mathcal{O}_{j}^{(1)} \mathcal{O}_{i}^{(2)}\right\rangle_{Y_{4}}=\lambda^{i}\left\langle\mathcal{O}_{j}^{(1)} \mathcal{O}_{i}^{(2)}\right\rangle_{Y_{3}}=\lambda^{i} \eta_{j i}^{(1)}
$$

As already mentioned above, two-point functions receive no worldsheet instanton corrections and the classical part of the amplitude is already the exact expression. Integrating twice we obtain the term in (52) which does not contain instanton corrections.

Finally, the contribution to the superpotential which has no component in the base is subleading in the limit where $\lambda^{V i}$ and $t^{V}$ are large. To summarize, we confirmed the expression given in eq. (52) by considering correlation functions in the topological A-model, that is without using mirror symmetry. Altogether we thus have

$$
\tilde{W}\left(Y_{4}\right)=\lambda^{k} \eta_{j k}^{(1)} t^{j} t^{V}+\lambda^{V k} \mathcal{F}_{k}
$$

with the relations $\lambda^{k} \eta_{j k}^{(1)}=\nu_{j}, \lambda^{V k}=-\tilde{\mu}^{k}$. 


\subsection{The superpotential for $K 3$-fibred fourfolds}

In this section we further specify to $K 3$-fibred fourfolds so that we are able to use the type IIA - heterotic duality and derive the heterotic superpotential including the type IIA quantum corrections. Specifically we choose the fourfold to be $K 3$-fibred over a large base $\mathbb{F}_{n}$ as we did in section 2.3. Such fourfolds can also be viewed as $Y_{3}$ fibred fourfolds over a base $\mathbb{P}_{1}$ where the $Y_{3}$-fibre is itself $K 3$-fibred over a second $\mathbb{P}_{1}$. This allows us to make contact with the results of the last section and merely specify them for $K 3$-fibred threefolds.

The case of $K 3$-fibred threefolds has been studied in detail in connection with the heterotic - type IIA duality for $N=2, D=4$ string vacua [25]. For such threefolds the prepotential $\mathcal{F}$ obeys in the large $\mathbb{P}_{1}$ limit

$$
\mathcal{F}=t^{U} \eta_{\hat{\imath} \hat{\jmath}} t^{\hat{\imath}} t^{\hat{\jmath}}+\mathcal{F}^{(1)}\left(t^{\hat{\imath}}, t^{\hat{\jmath}}\right)+\mathcal{O}\left(e^{i t^{U}}\right),
$$

where $t^{U}$ is the modulus parameterizing the $\mathbb{P}_{1}$ base while the $t^{\hat{\imath}}$ denote the moduli of the $K 3$-fibre except those from reducible bad fibres. $\left(\eta_{\hat{\imath} \hat{\jmath}}\right.$ has been defined in (21).)

Inserting (66) into (44) one obtains the superpotential for $K 3$-fibred fourfolds in the large $\mathbb{F}_{n}$ limit. If one assumes that the duality map given in (26) continues to hold in the presence of background fluxes one can use it to derive a heterotic superpotential $W_{\text {het }}\left(\tau, \rho, n^{a}, u, v\right)$. However, this might be possible only for a subset of the fluxes considered so far [36].

As we discussed in section 2.3 both the heterotic vacua and the dual type IIA vacua enjoy a natural action of the symmetry group $S L(2, \mathbb{Z}) \times S L(2, \mathbb{Z})$. This is a subgroup of the symplectic $S p\left(2 h^{(1,1)}+2\right)$ symmetry group which we discussed in section 3.2.1. More concretely the transformation (13) acts as a specific symplectic transformation (49) on the homogeneous coordinates corresponding to $\left(\rho, \tau, n^{a}, u\right)$ respectively $t^{i}$, which in the large base limit leaves $u$ and $t^{U}$ invariant [37]. From the discussion of section 3.2.1 it is clear that the $S L(2, \mathbb{Z}) \times S L(2, \mathbb{Z})$ transformations leave both the Kähler potential and the superpotential invariant if they are expressed in terms of homogeneous coordinates. In special coordinates the $S L(2, \mathbb{Z}) \times S L(2, \mathbb{Z})$ transformations instead lead to a Kähler transformation of the Kähler- and the superpotential which also leaves the action invariant.

Finally we remark that the heterotic origin of some of the background fluxes $F_{2}$ and $F_{4}$ might be understood from the duality between the heterotic string on $T^{4}$ and the type IIA theory on $K 3$, as was first noted in [12]. For example, denoting by $e_{U / V}$ the two $(1,1)$-forms stemming from the base $\mathbb{F}_{n}$ and by $e_{\hat{\imath}}$ those of the fibre $K 3$, a background $F_{4} \sim \lambda^{U / V \hat{\imath}} e_{U / V} \wedge e_{\hat{\imath}}$ can be interpreted as giving a background flux along $e_{U / V}$ to the field strength of the $D=6$ vectors coming from expanding $A_{3}$ in terms of the $e_{\hat{\imath}}$. These vectors have heterotic counterparts $A^{\hat{\imath}}$ according to the $D=6$ duality. Expanding their field strength as $F_{2}^{\hat{\imath}} \sim \lambda^{U / V \hat{\imath}} e_{U / V}$ should correspond to the above background $F_{4}$. A similar situation holds for $F_{4} \sim \lambda^{U V} e_{U} \wedge e_{V}$ and $F_{2} \sim \lambda^{U / V} e_{U / V}$, but there seems to be no such simple heterotic correspondence for all other background fluxes. Some of them might be related to non-vanishing torsion on the heterotic side [8]. 


\section{Acknowledgements}

We thank A. Klemm, P. Mayr, H. Nicolai and H. Singh for usefull conversations and correspondence. We also thank the authors of ref. 34] for communicating their results prior to publication.

This work is supported by DFG (the German Science Foundation), GIF (the GermanIsraeli Foundation for Scientific Research), DAAD (the German Academic Exchange Service) and the Landesgraduiertenförderung Sachsen-Anhalt.

\section{Appendix}

\section{A Notation}

The signature of the space-time metric is $(-+\ldots+)$. The Levi-Civita symbol is defined to transform as a tensor, i.e. we have

$$
\epsilon^{1 \ldots D}=\left( \pm g^{(D)}\right)^{-1 / 2} \quad \text { and } \quad \epsilon_{1 \ldots D}= \pm\left( \pm g^{(D)}\right)^{1 / 2}
$$

where the + sign corresponds to Euclidean and the - sign to Minkowskian signature. Our conventions for the Riemann curvature tensor are

$$
R_{\nu \rho \sigma}^{\mu}=\partial_{\rho} \Gamma_{\nu \sigma}^{\mu}-\partial_{\sigma} \Gamma_{\nu \rho}^{\mu}+\Gamma_{\nu \sigma}^{\omega} \Gamma_{\omega \rho}^{\mu}-\Gamma_{\nu \rho}^{\omega} \Gamma_{\omega \sigma}^{\mu}
$$

where we use the following definition of the Christoffel symbols:

$$
\Gamma_{\nu \rho}^{\mu}=\frac{1}{2} g^{\mu \sigma}\left(\partial_{\nu} g_{\sigma \rho}+\partial_{\rho} g_{\sigma \nu}-\partial_{\sigma} g_{\nu \rho}\right)
$$

The Ricci tensor is defined as

$$
R_{\mu \nu}=R_{\mu \rho \nu}^{\rho} .
$$

We are thus using the $(+++)$ conventions of 38 .

Furthermore a $p$-form $A_{p}$ can be expanded as

$$
A_{p}=\frac{1}{p !} A_{\mu_{1} \ldots \mu_{p}} d x^{\mu_{1}} \wedge \ldots \wedge d x^{\mu_{p}}
$$

which has an obvious generalisation to $(p, q)$-forms:

$$
A_{p, q}=\frac{1}{p ! q !} A_{i_{1} \ldots i_{p} \bar{\imath}_{1} \ldots \bar{\nu}_{q}} d \xi^{i_{1}} \wedge \ldots \wedge d \xi^{i_{p}} \wedge d \bar{\xi}^{\overline{1}_{1}} \wedge \ldots \wedge d \bar{\xi}^{\bar{\eta}_{q}}
$$

The exterior derivative is defined as

$$
d A_{p}=\frac{1}{p !} \partial_{\mu} A_{\mu_{1} \ldots \mu_{p}} d x^{\mu} \wedge d x^{\mu_{1}} \wedge \ldots \wedge d x^{\mu_{p}},
$$

which entails because of

$$
F_{p+1}=d A_{p}=\frac{1}{(p+1) !} F_{\mu_{1} \ldots \mu_{p+1}} d x^{\mu_{1}} \wedge \ldots \wedge d x^{\mu_{p+1}}
$$


the relation

$$
F_{\mu_{1} \ldots \mu_{p+1}}=(p+1) \partial_{\left[\mu_{1}\right.} A_{\left.\mu_{2} \ldots \mu_{p+1}\right]} .
$$

With this definition the action for a $p$-form potential $A_{p}$ is given by

$$
-\frac{1}{4} \int d^{D} x \sqrt{-g^{(D)}}\left|F_{p+1}\right|^{2}=-\frac{1}{4} \int d^{D} x \frac{\sqrt{-g^{(D)}}}{(p+1) !} F_{\mu_{1} \ldots \mu_{p+1}} F^{\mu_{1} \ldots \mu_{p+1}} .
$$

The Hodge star operator for a $p$-form is defined as

$$
\star A_{p}=\frac{1}{p !(D-p) !} A_{\mu_{1} \ldots \mu_{p}} \epsilon_{\nu_{p+1} \ldots \nu_{D}}^{\mu_{1} \ldots \mu_{p}} d x^{\nu_{p+1}} \wedge \ldots \wedge d x^{\nu_{D}} .
$$

It is generalised to $(p, q)$-forms on a Hermitian manifold (where $D$ now denotes its complex dimension) by

$$
\begin{aligned}
\star A_{p, q}=\frac{(-1)^{(D-p) q+1 / 2 D(D-1)} i^{D}}{p ! q !(D-p) !(D-q) !} & \bar{A}_{i_{1} \ldots i_{q} \bar{\imath}_{1} \ldots \bar{\nu}_{p}} \epsilon^{i_{1} \ldots i_{q}} \bar{\jmath}_{q+1} \ldots \bar{\jmath}_{D} \epsilon^{\bar{\imath}_{1} \ldots \bar{\imath}_{p}}{ }_{j_{p+1} \ldots j_{D}} \\
\times d \xi^{j_{p+1}} & \wedge \ldots \wedge d \xi^{j_{D}} \wedge d \bar{\xi}^{\bar{\jmath}^{\bar{j}_{q+1}}} \wedge \ldots \wedge d \bar{\xi}^{\bar{\xi}_{D}}
\end{aligned}
$$

where $\bar{A}_{i_{1} \ldots i_{q} \bar{\tau}_{1} \ldots \bar{z}_{p}}=\overline{A_{i_{1} \ldots i_{p} \bar{\imath}_{1} \ldots \bar{q}_{q}}}$. This definition ensures that we have the following expression for the scalar product of two $(p, q)$-forms on a Hermitian manifold:

$$
\begin{aligned}
\left(A_{p, q}, B_{p, q}\right) & \equiv \int A_{p, q} \wedge \star B_{p, q} \\
& =\frac{(-1)^{1 / 2 D(D-1)} i^{D}}{p ! q !} \int \sqrt{g^{(D)}} A_{i_{1} \ldots i_{p} \bar{\imath}_{1} \ldots \bar{\nu}_{q}} \bar{B}^{\bar{p}_{1} \ldots \bar{\imath}_{q} i_{1} \ldots i_{p}} d^{D} \xi d^{D} \bar{\xi}
\end{aligned}
$$

\section{B $T^{2}$ compactification of $D=4$ supergravity}

In this appendix we give some details of the $T^{2}$ reduction of the $D=4, N=1$ supergravity given in equation (11). As already mentioned in the main text there are two equivalent ways to derive the effective action in $D=2$. One can either directly compactify the $D=4$ action on a torus. This leads naturally to the variables relevant for the purpose of this paper. One can also perform the reduction in two steps with the detour over $D=3$. For completeness we give this derivation too. In this case one can make use of the results given in [22].

Let us first perform the direct torus-reduction. In contrast to the main text we here work in the Einstein-frame because this is easier to handle in the general case where one does not specify the Kähler potential and gauge kinetic function to their tree level form. Inserting (3) and (4) (but now in the Einstein-frame) into (1), using the definitions (5), (7) and (8) one derives the following $D=2$ effective Lagrangian

$$
\begin{aligned}
\mathcal{L}_{\text {het }}^{(2)}= & \sqrt{h_{(E)}}\left(-\frac{1}{2} \partial_{\mu} \partial^{\mu} \sigma+\frac{\partial_{\mu} \tau \partial^{\mu} \bar{\tau}}{(\tau-\bar{\tau})^{2}}-G_{I \bar{J}}^{(4)} \partial_{\mu} \Phi^{I} \partial^{\mu} \bar{\Phi}^{\bar{J}}\right) \\
& -(\tau-\bar{\tau})^{-1}\left(i\left(\operatorname{Re} f_{a b}\right) D_{\mu} n^{a} D^{\mu} \bar{n}^{b}-\frac{1}{2} \epsilon^{\mu \nu}\left(\partial_{\mu} \operatorname{Im} f_{a b}\right)\left(\bar{n}^{a} D_{\nu} n^{b}-n^{a} D_{\nu} \bar{n}^{b}\right)\right)
\end{aligned}
$$


where $h_{(E)}$ denotes the determinant of the torus metric in the Einstein-frame and we have chosen the conformal gauge for the two-dimensional space-time metric

$$
g_{\mu \nu}^{(2)}=e^{\sigma} h_{(E)}^{1 / 4} \eta_{\mu \nu}
$$

As in equation (11) the $\Phi^{I}$ are all moduli of the $D=4$ Lagrangian including the dilaton $S$. Furthermore we have omitted all vectors because they do not have dynamical degrees of freedom in $D=2$.

As has been shown in [20] the moduli space of non-linear $(2,2)$ sigma-models in $D=2$ is in general not Kähler, when chiral and twisted chiral multiplets are present. Nevertheless the Lagrangian can be expressed by second-order partial derivatives of a real function of the moduli, analogous to the Kähler potential of a Kähler manifold. In case of dilaton-supergravity the general form of the Lagrangian has been given in [26]. In our case $\sqrt{h_{(E)}}$ takes the role of the $D=2$ dilaton. 1 The Lagrangian (B.1) is the sum of two terms, one multiplied by $\sqrt{h_{(E)}}$, the other one not. Both terms can seperately be expressed via a potential.

Defining the two real functions

$$
\begin{aligned}
K_{1}^{\text {het }} & =K^{(4)}(\Phi, \bar{\Phi})+\ln (-i(\tau-\bar{\tau})) \\
K_{2}^{\text {het }} & =\frac{i}{2}\left(\operatorname{Re} f_{a b}(\Phi)\right)\left[(\tau-\bar{\tau})^{-1}\left(n^{a}-\bar{n}^{a}\right)\left(n^{b}-\bar{n}^{b}\right)\right]
\end{aligned}
$$

and denoting collectively $\left(\tau, n^{a}\right)$ as $\chi^{\Sigma}$ we can express (B.1) in the form

$$
\begin{aligned}
\mathcal{L}_{\text {het }}^{(2)}= & \sqrt{h_{(E)}}\left(-\frac{1}{2} \partial_{\mu} \partial^{\mu} \sigma-\left(\partial_{\Phi^{I}} \bar{\partial}_{\bar{\Phi}^{\bar{J}}} K_{1}^{\text {het }}\right) \partial_{\mu} \Phi^{I} \partial^{\mu} \bar{\Phi}^{\bar{J}}+\left(\partial_{\chi^{\Sigma}} \bar{\partial}_{\bar{\chi}^{\bar{\Lambda}}} K_{1}^{\text {het }}\right) \partial_{\mu} \chi^{\Sigma} \partial^{\mu} \bar{\chi}^{\bar{\Lambda}}\right) \\
& +\left(\partial_{\chi^{\Sigma}} \bar{\partial}_{\bar{\chi}^{\bar{\Lambda}}} K_{2}^{\text {het }}\right) \partial_{\mu} \chi^{\Sigma} \partial^{\mu} \bar{\chi}^{\bar{\Lambda}} \\
& -\epsilon^{\mu \nu}\left(\bar{\partial}_{\bar{\Phi}^{\bar{J}}} \partial_{\chi^{\Sigma}} K_{2}^{\text {het }}\right) \partial_{\mu} \bar{\Phi}^{\bar{J}} \partial_{\nu} \chi^{\Sigma}-\epsilon^{\mu \nu}\left(\partial_{\Phi^{I}} \bar{\partial}_{\bar{\chi}^{\bar{\Lambda}}} K_{2}^{\text {het }}\right) \partial_{\mu} \Phi^{I} \partial_{\nu} \bar{\chi}^{\bar{\Lambda}} .
\end{aligned}
$$

This is of the form given in [20, 26]. Obviously the moduli space is not Kähler. As explained in 20] the terms proportional to $\epsilon^{\mu \nu}$ always combine a derivative with respect to a chiral field with a derivative with respect to a twisted chiral field. The $\Phi^{I}$ stem from the chiral $D=4$ moduli and continue to be chiral in $D=2$. In view of the last line in (B.5) this means that $\left(\tau, n^{a}\right)$ reside in twisted chiral multiplets.

In the alternative derivation of the $D=2$ effective action one reduces the $D=3$ effective action obtained in 22] on a further circle. Recall that in $D=3$ an Abelian vector is dual to a scalar and thus a vector multiplet can be dualized to a scalar multiplet. Hence the three-dimensional Lagrangian on the Coulomb branch can be entirely expressed in terms of chiral multiplets. The bosonic part of this Lagrangian is given in the Einsteinframe by

$$
\mathcal{L}_{\text {het }}^{(3)}=\sqrt{g^{(3)}}\left(\frac{1}{2} R^{(3)}-G_{\bar{\Lambda} \Sigma} \partial_{m} \bar{Z}^{\bar{\Lambda}} \partial^{m} Z^{\Sigma}\right), \quad m=0,1,2,
$$

where $Z^{\Sigma}$ are the complex scalar fields in the three-dimensional chiral multiplets. They contain the moduli of the four-dimensional heterotic theory $\Phi^{I}$, the radius and the

\footnotetext{
${ }^{18}$ In fact $\sqrt{h_{(E)}}$ is the heterotic $D=2$ dilaton because $e^{-2 \Phi_{\text {het }}^{(2)}}=e^{-2 \Phi_{\text {het }}^{(4)}} \sqrt{h}=\sqrt{h_{(E)}}$. The last equality is due to the Weyl rescaling relating the $D=4$ Einstein- and string-frame metrics.
} 
Kaluza-Klein gauge boson of $S^{1}$ combined into a complex scalar $T$ and finally the scalars $D^{a}$ which reside in the chiral multiplets dual to the vector multiplets. [9 Hence we have a decomposition $Z^{\Sigma}=\left(\Phi^{I}, T, D^{a}\right)$. Supersymmetry constrains the metric $G_{\bar{\Lambda} \Sigma}$ to be Kähler, $G_{\bar{\Lambda} \Sigma}=\bar{\partial}_{\bar{\Lambda}} \partial_{\Sigma} K_{\text {het }}^{(3)}$, and in terms of the scalars $Z^{\Sigma}$ the Kähler potential reads

$$
K_{\text {het }}^{(3)}=K_{\text {het }}^{(4)}(\Phi, \bar{\Phi})-\ln \left[-i(T-\bar{T})+\frac{1}{2}(D-\bar{D})^{a}(\operatorname{Re} f)_{a b}^{-1}(\Phi)(D-\bar{D})^{b}\right] .
$$

$\mathcal{L}_{\text {het }}^{(3)}$ can be further reduced on a second $S^{1}$ using the Ansatz

$$
g_{m n}^{(3)}=\left(\begin{array}{cc}
g_{\mu \nu}^{(2)} & 0 \\
0 & r^{2}
\end{array}\right)
$$

where $\mu, \nu=0,1$ and $r$ is the radius of the $S^{1}$ measured in the $D=3$ Einstein-frame metric. There are no new Kaluza-Klein gauge bosons in this reduction since they contain no physical degree of freedom. Inserting (B.8) into (B.6) results in

$$
\mathcal{L}_{\text {het }}^{(2)}=\sqrt{g^{(2)}} r\left[\frac{1}{2} R^{(2)}-G_{\bar{\Lambda} \Sigma} \partial_{\mu} \bar{Z}^{\bar{\Lambda}} \partial^{\mu} Z^{\Sigma}\right] .
$$

Choosing the conformal gauge

$$
g_{\mu \nu}^{(2)}=e^{\sigma} \eta_{\mu \nu},
$$

and using the relation $r=e^{-2 \Phi_{\text {het }}^{(2)}}$ between the radius of the circle measured in the $D=3$ Einstein-frame metric and the $D=2$ heterotic dilaton defined in equation (7) one derives

$$
\mathcal{L}_{\text {het }}^{(2)}=e^{-2 \Phi_{\text {het }}^{(2)}}\left[-\frac{1}{2} \partial_{\mu} \partial^{\mu} \sigma-G_{\bar{\Lambda} \Sigma} \partial_{\mu} \bar{Z}^{\bar{\Lambda}} \partial^{\mu} Z^{\Sigma}\right] .
$$

The physical degrees of freedom are exactly the same as in $D=3$. Also the fact that all scalars $Z^{\Sigma}$ are members of chiral multiplets is inherited from $D=3$. Thus in contrast to equation (B.5) no twisted chiral multiplets occur and the moduli space is therefore a Kähler manifold. In fact the sigma-model geometry is unchanged in the reduction from $D=3$ to $D=2$, that is $G_{\bar{\Lambda} \Sigma}$ is the same Kähler metric with the same Kähler potential as in $D=3$.

\section{Dimensional reduction of type IIA supergravity on Calabi-Yau fourfolds}

In this appendix we present some of the details of the dimensional reduction of the type IIA supergravity Lagrangian (14) on Calabi-Yau fourfolds including the $(2,1)$-moduli which have been neglected in the main text. We follow closely the procedure applied in ref. [39, 22] for compactification of type IIA on Calabi-Yau threefolds. Starting from the Ansatz

$$
g_{M N}^{(10)}(x, y)=\left(\begin{array}{cc}
g_{\mu \nu}^{(2)}(x) & 0 \\
0 & g_{a b}^{(8)}(x, y)
\end{array}\right),
$$

\footnotetext{
${ }^{19}$ For the exact definition of the fields $T$ and $D^{a}$ see 22 . Note however, that we performed a redefinition $T, D^{a} \rightarrow i T, i D^{a}$ here.
} 
for the $\mathrm{D}=10$ space-time metric one considers infinitesimally small deformations of the metric

$$
g_{a b}^{(8)}=\hat{g}_{a b}^{(8)}(\langle M\rangle)+\delta g_{a b}^{(8)}(\langle M\rangle, \delta M(x))
$$

where $\hat{g}_{a b}^{(8)}$ is a background metric and $\delta g_{a b}^{(8)}$ its deformation. Demanding that $\delta g_{a b}^{(8)}$ preserves the Calabi-Yau condition one can expand it in terms of non-trivial harmonic forms on $Y_{4}$. It is convenient to introduce complex coordinates $\xi_{j}(j=1, \ldots, 4)$ for $Y_{4}$ defining $\xi_{j}=\frac{1}{\sqrt{2}}\left(y_{2 j-1}+i y_{2 j}\right)$. For the deformation of the Kähler form one has ${ }^{20}$

$$
i \delta g_{i \bar{\jmath}}=\sum_{A=1}^{h^{1,1}} \delta M^{A}(x) e_{i \bar{\jmath}}^{A}
$$

where $e^{A}$ is an appropriate basis of $H^{1,1}\left(Y_{4}\right)$ and $M^{A}(x)$ are the corresponding real moduli. For the deformations of the complex structure one has

$$
\delta g_{\bar{\imath} \bar{\jmath}}=\sum_{\alpha=1}^{h^{3,1}} \delta Z^{\alpha}(x) b_{\bar{\imath} \bar{\jmath}}^{\alpha}
$$

where $Z^{\alpha}(x)$ are complex moduli and $b_{\bar{\imath} \bar{j}}^{\alpha}$ is related to the basis $\Phi^{\alpha}$ of $H^{3,1}\left(Y_{4}\right)$ by an appropriate contraction with the anti-holomorphic 4-form $\bar{\Omega}$ on $Y_{4}$ 40:

$$
b_{\bar{\imath} \bar{\jmath}}^{\alpha}=-\frac{1}{3|\Omega|^{2}} \bar{\Omega}_{\bar{\imath}}^{k l m} \Phi_{k l m \bar{\jmath}}^{\alpha}, \quad|\Omega|^{2} \equiv \frac{1}{4 !} \Omega_{i j k l} \bar{\Omega}^{i j k l}
$$

The 3 -form $C_{3}$ is expanded in terms of the (2,1)-forms $\Psi_{i j \bar{k}}^{I}$. More precisely

$$
C_{i j \bar{k}}=\sum_{I=1}^{h^{2,1}} N^{I}(x) \Psi_{i j \bar{k}}^{I}, \quad C_{\bar{\imath} \bar{\jmath} k}=\sum_{I=1}^{h^{2,1}} \bar{N}^{\bar{J}}(x) \bar{\Psi}_{\bar{\imath} \bar{\jmath} k}^{\bar{J}} .
$$

Finally the antisymmetric tensor is expanded in terms of the $(1,1)$-forms $e^{A}$ according to

$$
B_{i \bar{\jmath}}=\sum_{A=1}^{h^{1,1}} a^{A}(x) e_{i \bar{\jmath}}^{A}
$$

Again the vectors are neglected because they do not propagate in $D=2$.

The dimensional reduction is performed by inserting (C.1), (C.6) and (C.7) into (14). One has to take into account, that the $(2,1)$-forms depend on the complex structure of the Calabi-Yau. The basis $\Psi^{I}$ of $(2,1)$-forms can locally be chosen to depend holomorphically on the complex structure or in other words

$$
\partial_{\bar{Z}^{\bar{\alpha}}} \Psi^{I}=0, \quad \partial_{Z^{\alpha}} \Psi^{I} \neq 0
$$

The derivative $\partial_{Z^{\alpha}} \Psi^{I}$ can be expanded into $(1,2)$ - and $(2,1)$-forms with complex-structure dependent coefficient functions $\sigma$ and $\tau$

$$
\partial_{Z^{\alpha}} \Psi^{I}=\sigma_{\alpha I K}(Z, \bar{Z}) \Psi^{K}+\tau_{\alpha I \bar{L}}(Z, \bar{Z}) \bar{\Psi}^{\bar{L}}
$$

\footnotetext{
${ }^{20}$ In the following we omit the superscript (8) at the internal metric.
} 
We define a metric $G_{I \bar{J}}$ and intersection numbers $d_{A I \bar{J}}$ on the space of $(2,1)$-forms

$$
\begin{aligned}
G_{I \bar{J}} & \equiv \frac{1}{4} \int_{Y_{4}} \Psi_{I} \wedge \star \Psi_{J} \\
d_{A I \bar{J}} & \equiv \int_{Y_{4}} e_{A} \wedge \Psi_{I} \wedge \bar{\Psi}_{\bar{J}} .
\end{aligned}
$$

They are related via 22

$$
G_{I \bar{J}}=-\frac{i}{2} d_{A I \bar{J}} M^{A}
$$

Because of (C.9) they depend on the complex structure moduli $Z^{\alpha}$. Using (C.10) and (17) the dimensional reduction of (14) yields

$$
\begin{aligned}
\mathcal{L}_{\mathrm{IIA}}^{(2)} & =\sqrt{-g^{(2)}} e^{-2 \Phi_{\mathrm{IIA}}^{(2)}}\left(\frac{1}{2} R^{(2)}+2 \partial_{\mu} \Phi_{\mathrm{IIA}}^{(2)} \partial^{\mu} \Phi_{\mathrm{IIA}}^{(2)}-G_{A \bar{B}} \partial_{\mu} t^{A} \partial^{\mu} \bar{t}^{\bar{B}}-G_{\alpha \bar{\beta}} \partial_{\mu} Z^{\alpha} \partial^{\mu} \bar{Z}^{\bar{\beta}}\right) \\
& -\sqrt{-g^{(2)}}\left(G_{I \bar{J}} D_{\mu} N^{I} D^{\mu} \bar{N}^{\bar{J}}+\frac{1}{4} d_{A I \bar{J}} \epsilon^{\mu \nu} \partial_{\mu} a^{A}\left[N^{I} D_{\nu} \bar{N}^{\bar{J}}-\bar{N}^{\bar{J}} D_{\nu} N^{I}\right]\right),(\mathrm{C} .12)
\end{aligned}
$$

where

$$
D_{\mu} N^{I}=\partial_{\mu} N^{I}+N^{K} \sigma_{\alpha K I}(Z, \bar{Z}) \partial_{\mu} Z^{\alpha}+\bar{N}^{\bar{L}} \bar{\tau}_{\bar{\beta} \bar{L} I}(Z, \bar{Z}) \partial_{\mu} \bar{Z}^{\bar{\beta}}
$$

As in the heterotic case there are two terms, one has a factor $e^{-2 \Phi_{\text {IIA }}^{(2)}}$ and the other one not. Again both can seperately be expressed via a potential. We define

$$
\begin{aligned}
K_{1}^{\mathrm{IIA}} & =-\ln \left[\int_{Y_{4}} \Omega \wedge \bar{\Omega}\right]+\ln \mathcal{V}, \\
K_{2}^{\mathrm{IIA}} & =\frac{i}{\sqrt{2}}\left(t^{A}-\bar{t}^{A}\right)\left(\frac{i}{2} d_{A M \bar{L}} \hat{G}_{\bar{J} M}^{-1} \hat{G}_{\bar{L} I}^{-1} \hat{N}^{I} \overline{\hat{N}}^{\bar{J}}-\omega_{A I K} \hat{N}^{I} \hat{N}^{K}-\bar{\omega}_{A \bar{J} \bar{L}} \overline{\hat{N}}^{\bar{J}} \overline{\hat{N}}^{\bar{L}}\right),
\end{aligned}
$$

where we have introduced new coordinates

$$
\hat{N}^{I}=\hat{G}_{I \bar{J}}(Z, \bar{Z}) \bar{N}^{\bar{J}}
$$

with

$$
\hat{G}_{I \bar{J}}=-\frac{i}{2} d_{A I \bar{J}} c^{A}
$$

and $c^{A}$ is a constant nonzero vector. The $\omega_{A I K}$ are functions of $Z^{\alpha}$ and $\bar{Z}^{\bar{\alpha}}$ which have to obey

$$
\partial_{\bar{Z}^{\bar{\alpha}} \omega_{A I K}}=-\frac{i}{4} \hat{G}_{\bar{L} I}^{-1} \hat{G}_{\bar{J} K}^{-1} d_{A M \bar{L}} \bar{\tau}_{\bar{\alpha} \bar{J} M}
$$

but are otherwise unconstrained.

With the help of $K_{1}^{\mathrm{IIA}}$ and $K_{2}^{\mathrm{IIA}}$ and denoting collectively the fields $\left(Z^{\alpha}, \hat{N}^{I}\right)$ as $\Phi^{\Sigma}$ the Lagrangian (C.12) can be expressed as

$$
\begin{aligned}
\mathcal{L}_{\mathrm{IIA}}^{(2)}= & e^{-2 \Phi_{\mathrm{IIA}}^{(2)}}\left(-\frac{1}{2} \partial_{\mu} \partial^{\mu} \sigma-\left(\partial_{\Phi^{\Sigma}} \bar{\partial}_{\bar{\Phi}^{\bar{\Lambda}}} K_{1}^{\mathrm{IIA}}\right) \partial_{\mu} \Phi^{\Sigma} \partial^{\mu} \bar{\Phi}^{\bar{\Lambda}}+\left(\partial_{t^{A}} \bar{\partial}_{\bar{t}_{\bar{B}}} K_{1}^{\mathrm{IIA}}\right) \partial_{\mu} t^{A} \partial^{\mu} \bar{t}^{\bar{B}}\right) \\
& -\left(\partial_{\Phi^{\Sigma}} \bar{\partial}_{\bar{\Phi}_{\bar{\Lambda}}} K_{2}^{\mathrm{IIA}}\right) \partial_{\mu} \Phi^{\Sigma} \partial^{\mu} \bar{\Phi}^{\bar{\Lambda}}+\epsilon^{\mu \nu}\left(\bar{\partial}_{\bar{\Phi}_{\bar{\Lambda}}} \partial_{t^{A}} K_{2}^{\mathrm{IIA}}\right) \partial_{\mu} \bar{\Phi}^{\bar{\Lambda}} \partial_{\nu} t^{A} \\
& +\epsilon^{\mu \nu}\left(\partial_{\Phi^{\Lambda}} \bar{\partial}_{\bar{t}_{\bar{A}}} K_{2}^{\mathrm{IIA}}\right) \partial_{\mu} \Phi^{\Lambda} \partial_{\nu} \bar{t}^{\bar{A}}
\end{aligned}
$$


where the conformal gauge has been chosen

$$
g_{\mu \nu}^{(2)}=e^{\sigma} e^{2 \Phi_{\mathrm{IIA}}^{(2)}} \eta_{\mu \nu}
$$

This is of the form given in 20, 26] and the terms proportional to $\epsilon^{\mu \nu}$ display the fact that the $\Phi^{\Sigma}$ reside in chiral multiplets whereas the $t^{A}$ are members of twisted chiral multiplets. These are the coordinates which are relevant for this paper.

It is however possible to express the $D=2$ effective action (C.12) by chiral multiplets only. For this purpose it is necessary to dualise the scalars $a^{A}$. This is possible, because they only appear via their 'field strength' $\partial_{\mu} a^{A}$ in (C.12). One adds $-F_{\mu}^{A} \partial^{\mu} P^{A}$ to (C.12), where $P^{A}$ is a Lagrange multiplier and $F^{A \rho} \equiv \epsilon^{\rho \mu} \partial_{\mu} a^{A}$. Then one eliminates $F^{A}$ via its equation of motion in favour of $P^{A}$. Rescaling the $M^{A}$ according to

$$
\tilde{M}^{A}=e^{-2 / 3 \Phi_{\mathrm{IIA}}^{(10)}} M^{A}
$$

rescales the couplings as follows

$$
\begin{aligned}
\tilde{G}_{A B} & =G_{A B} e^{4 / 3 \Phi_{\mathrm{IIA}}^{(10)}} \\
\tilde{G}_{I \bar{J}} & =G_{I \bar{J}} e^{-2 / 3 \Phi_{\mathrm{IIA}}^{(10)}} \\
\tilde{\mathcal{V}} & =e^{-8 / 3 \Phi_{\mathrm{IIA}}^{(10)}} \mathcal{V}=e^{-2 / 3 \Phi_{\mathrm{IIA}}^{(10)}} e^{-2 \Phi_{\mathrm{IIA}}^{(2)}} .
\end{aligned}
$$

Finally, we choose a different conformal gauge for the two-dimensional metric

$$
g_{\mu \nu}^{(2)}=e^{2 \Phi_{\mathrm{IIA}}^{(10)}} e^{4 \Phi_{\mathrm{IIA}}^{(2)}} e^{\sigma} \eta_{\mu \nu}
$$

Inserting these field redefinitions into (C.12) and performing the duality transformation yields

$$
\begin{aligned}
\mathcal{L}_{I I A}^{(2)}= & e^{-2 \Phi_{\mathrm{IIA}}^{(2)}}\left[-\frac{1}{2} \partial_{\mu} \partial^{\mu} \sigma-G_{\alpha \bar{\beta}} \partial_{\mu} Z^{\alpha} \partial^{\mu} \bar{Z}^{\bar{\beta}}-\tilde{\mathcal{V}}^{-1} \tilde{G}_{I \bar{J}} D_{\mu} N^{I} D^{\mu} \bar{N}^{\bar{J}}\right. \\
& -\frac{1}{2} \partial_{\mu} \ln \tilde{\mathcal{V}} \partial^{\mu} \ln \tilde{\mathcal{V}}-\frac{1}{2} \tilde{G}_{A B} \partial_{\mu} \tilde{M}^{A} \partial^{\mu} \tilde{M}^{B} \\
& -\frac{1}{2 \tilde{\mathcal{V}}^{2}}\left(\partial_{\mu} P^{A}+\frac{1}{4} d_{A K \bar{L}}\left(N^{K} D_{\mu} \bar{N}^{\bar{L}}-D_{\mu} N^{K} \bar{N}^{\bar{L}}\right)\right) \\
& \left.\tilde{G}_{A B}^{-1}\left(\partial^{\mu} P^{B}+\frac{1}{4} d_{B I \bar{J}}\left(N^{I} D^{\mu} \bar{N}^{\bar{J}}-D^{\mu} N^{I} \bar{N}^{\bar{J}}\right)\right)\right]
\end{aligned}
$$

This is exactly the effective action one gets by first reducing $D=11$ supergravity on the Calabi-Yau fourfold to $D=3$ as performed in [22] and then reducing on a further circle. To see this one has to use the relation between the $D=2$ type IIA dilaton and the radius $r$ of the circle measured in the $D=3$ Einstein-frame metric $r=e^{-2 \Phi_{\mathrm{IIA}}^{(2)}}$. In addition one has to choose the $D=2$ M-theory metric as

$$
g_{\mathrm{M} \mu \nu}^{(2)}=e^{\sigma} \eta_{\mu \nu}
$$

${ }^{21}$ The $\tilde{M}^{A}$ are precisely the Kähler moduli of M-theory used in [22]. 
It is clear from the discussion in [22] that one can write (C.23) in the form

$$
\mathcal{L}_{I I A}^{(2)}=e^{-2 \Phi_{\text {IIA }}^{(2)}}\left[-\frac{1}{2} \partial_{\mu} \partial^{\mu} \sigma-G_{\bar{\Lambda} \Sigma} \partial_{\mu} \bar{Z}^{\bar{\Lambda}} \partial^{\mu} Z^{\Sigma}\right]
$$

where $G_{\bar{\Lambda} \Sigma}=\bar{\partial}_{\bar{\Lambda}} \partial_{\Sigma} K_{\mathrm{M}}^{(3)}$ and $Z^{\Sigma}=\left\{T^{A}, \hat{N}^{I}, Z^{\alpha}\right\}$ with

$$
\begin{aligned}
K_{\mathrm{M}}^{(3)} & =-\ln \left[\int_{Y_{4}} \Omega \wedge \bar{\Omega}\right]-\ln \left[\Xi^{A} \mathcal{V} G_{A B}^{-1} \Xi^{B}\right] \\
\Xi^{A} & \equiv-i\left(T^{A}-\bar{T}^{A}-\frac{1}{2 \sqrt{8}} d_{A M \bar{L}} \hat{G}_{\bar{J} M}^{-1} \hat{G}_{\bar{L} I}^{-1} \hat{N}^{I} \overline{\hat{N}}^{\bar{J}}-\frac{i}{\sqrt{8}}\left(\omega_{A I K} \hat{N}^{I} \hat{N}^{K}+\bar{\omega}_{A \bar{J} \bar{L}} \overline{\hat{N}}^{\bar{J}} \overline{\hat{N}}^{\bar{L}}\right)\right) \\
T^{A} & =\frac{1}{\sqrt{8}}\left(-P^{A}+i \tilde{\mathcal{V}} \tilde{G}_{A B} \tilde{M}^{B}+\frac{1}{4} d_{A M \bar{L}} \hat{G}_{\bar{J} M}^{-1} \hat{G}_{\bar{L} I}^{-1} \hat{N}^{I} \overline{\hat{N}}^{\bar{J}}+i \omega_{A I K} \hat{N}^{I} \hat{N}^{K}\right)
\end{aligned}
$$

In the coordinates $Z^{\Sigma}$ the moduli space in $D=2$ is Kähler and has the same Kähler potential as in $D=3 .{ }^{2}$ Therefore the discussion of duality between the heterotic and type IIA theory in $D=2$ using the variables of (B.11) and (C.25) proceeds exactly along the same lines as in [22]. Finally comparing (C.25) with (B.11) shows

$$
e^{-2 \Phi_{\mathrm{het}}^{(2)}}=e^{-2 \Phi_{\mathrm{IIA}}^{(2)}} \text {. }
$$

This can also heuristically be derived from the duality between the heterotic and type IIA theory in $D=6$. This duality maps the string-frame metrics according to $g_{\text {het }}^{(6)}=e^{2 \Phi_{\text {het }}^{(6)}} g_{\text {IIA }}^{(6)}$ and the dilatons are related via $\Phi_{\text {het }}^{(6)}=-\Phi_{\text {IIA }}^{(6)}$. Starting from the effective action in $D=6$ and compactifying further on a four-dimensional manifold $B$ one derives

$$
\begin{aligned}
S & =\int d^{6} x \sqrt{-g_{\text {het }}^{(6)}} e^{-2 \Phi_{\text {het }}^{(6)}}\left(R_{\text {het }}^{(6)}+\ldots\right) \\
& =\int d^{2} x \sqrt{-g_{\text {het }}^{(2)}} e^{-2 \Phi_{\text {het }}^{(6)}} \mathcal{V}_{B}^{\text {het }}\left(R_{\text {het }}^{(2)}+\ldots\right) \\
& =\int d^{2} x \sqrt{-g_{\text {het }}^{(2)}} e^{-2 \Phi_{\text {het }}^{(2)}}\left(R_{\text {het }}^{(2)}+\ldots\right) .
\end{aligned}
$$

On the other hand the duality relations given above yield $e^{-2 \Phi_{\text {het }}^{(6)}} \mathcal{V}_{B}^{\text {het }}=e^{2 \Phi_{\text {het }}^{(6)}} \mathcal{V}_{B}^{\text {IIA }}=$ $e^{-2 \Phi_{\text {IIA }}^{(6)}} \mathcal{V}_{B}^{\text {IIA }}=e^{-2 \Phi_{\text {IIA }}^{(2)}}$, where all volumes are measured in the respective string-frame metrics.

\footnotetext{
${ }^{22}$ Note that in comparison with [22] we have here redefined the coordinates $T^{A} \rightarrow i T^{A}$.
} 


\section{References}

[1] For a review see, for example,

M. Green, J. Schwarz and E. Witten, Superstring theory, vol. 1\&2, Cambridge University Press, 1987;

J. Polchinski, String Theory, Cambridge University Press, 1998.

[2] C. Vafa, "Evidence for F Theory", Nucl. Phys. B469 (1996) 403, hep-th/9602022.

[3] S. Sethi, C. Vafa and E. Witten, "Constraints on Low-Dimensional String Compactification", Nucl. Phys. B480 (1996) 213, hep-th/9606122.

[4] P. Mayr, "Mirror Symmetry, $N=1$ Superpotentials and Tensionless Strings on Calabi-Yau Four-Folds", Nucl. Phys. B494 (1997) 489, hep-th/9610162.

[5] W. Lerche, "Fayet-Illiopoulos Potentials from Four-Folds", JHEP 9711 (1997) 004, hep-th/9709146.

[6] P. Kaste, "On the Twisted Chiral Potential in 2D and the Analogue of Rigid special Geometry for 4-Folds", JHEP 9906 (1999) 021, hep-th/9904218.

[7] S. Gukov, E. Witten and C. Vafa, "CFT's from Calabi-Yau Four-folds", Nucl. Phys. B584 (2000) 69, hep-th/9906070.

[8] K. Dasgupta, G. Rajesh and S. Sethi, "M-Theory, Orientifolds and G-Flux", JHEP 9908 (1999) 023, hep-th/9908088.

[9] S. Gukov, "Solitons, Superpotentials and Calibrations", Nucl. Phys. B574 (2000) 169, hep-th/9911011.

[10] B.R. Greene, K. Schalm and G. Shiu, "Warped Compactifications in M and F Theory", Nucl. Phys. B584 (2000) 480, hep-th/0004103.

[11] T. Taylor and C. Vafa, "RR Flux on Calabi-Yau and Partial Supersymmetry Breaking", Phys. Lett. B474 (2000) 130, hep-th/9912152.

[12] J. Polchinski and A. Strominger, "New Vacua for Type II String Theory", Phys. Lett. B388 (1996) 736, hep-th/9510227.

[13] J. Michelson, "Compactifications of Type IIB Strings to Four Dimensions with nontrivial Classical Potential", Nucl. Phys. B495 (1997) 127, hep-th/9610151.

[14] P. Mayr, "On Supersymmetry Breaking in String Theory and its Realization in Brane Worlds", hep-th/0003198.

[15] A. Ceresole, R. D'Auria, S. Ferrara and A. Van Proeyen, "Duality Transformations in Supersymmetric Yang-Mills Theories coupled to Supergravity", Nucl. Phys. B444 (1995) 92, hep-th/9502072.

[16] G.L. Cardoso, D. Lüst and T. Mohaupt, "Modular Symmetries of N=2 Black Holes", Phys. Lett. B388 (1996) 266, hep-th/9608099. 
[17] S. Ferrara, R. Kallosh and A. Strominger, " $N=2$ Extremal Black Holes", Phys. Rev. D52 (1995) 5412, hep-th/9508072;

S. Ferrara and R. Kallosh, "Supersymmetry and Attractors", Phys. Rev. D54 (1996) 1514, hep-th/9602136;

S. Ferrara and R. Kallosh, "Universality of Supersymmetric Attractors", Phys. Rev. D54 (1996) 1525, hep-th/9603090.

[18] For a recent review see, for example, T. Mohaupt, "Black Hole Entropy, Special Geometry and Strings", hep-th/0007195.

[19] S.J. Gates, M.T. Grisaru and M.E. Wehlau, "A Study of General 2D, N=2 Matter Coupled to Supergravity in Superspace", Nucl. Phys. B460 (1996) 579, hepth/9509021.

[20] S.J. Gates, C.M. Hull and M. Rocek, "Twisted Multiplets and New Supersymmetric Non-linear Sigma-Models", Nucl. Phys. B248 (1984) 157.

[21] B. de Wit, M.T. Grisaru, H. Nicolai and E. Rabinovici "Two-loop fFiniteness of D=2 Supergravity", Phys. Lett. B286 (1992) 78, hep-th/9205012.

[22] M. Haack and J. Louis, "Aspects of Heterotic/M-Theory Duality in $D=3$ ", hepth/9908067; "Duality in Heterotic Vacua with Four Supercharges", Nucl. Phys. B575 (2000) 107, hep-th/9912181.

[23] B.R. Greene, D.R. Morrison and M.R. Plesser, "Mirror Manifolds in Higher Dimension", Com. Math. Phys. 173 (1995) 559, hep-th/9402119.

[24] A. Klemm, B. Lian, S.S. Roan and S.T. Yau, "Calabi-Yau Fourfolds for M- and F-Theory Compactifications", Nucl. Phys. B518 (1998) 515, hep-th/9701023.

[25] For a review see, for example, K. Förger and J. Louis, "Holomorphic Couplings in String Theory", Nucl. Phys. Proc. Suppl. 55B (1997) 33, hep-th/9611184;

A. Klemm, "On the Geometry behind $N=2$ Supersymmetric Effective Actions in Four Dimensions", hep-th/9705131;

D. Lüst, "String Vacua with $\mathrm{N}=2$ Supersymmetry in Four Dimensions", hepth/9709222;

P. Aspinwall, "Compactification, Geometry and Duality: $N=2$ ", hep-th/0001001.

[26] S.J. Gates, S. Gukov and E. Witten, "Two Two-Dimensional Supergravity Theories from Calabi-Yau Four-Folds", Nucl. Phys. B584 (2000) 109, hep-th/0005120.

[27] E. Witten, "On Flux Quantization in M-Theory and the Effective Action", J. Geom. Phys. 22 (1997) 1, hep-th/9609122.

[28] M. Haack and J. Louis, work in progress.

[29] K. Becker and M. Becker, "M-Theory on Eight-Manifolds", Nucl. Phys. B477 (1996) 155, hep-th/9605053.

[30] H. Ooguri, Y. Oz and Z. Yin, "D-branes on Calabi-Yau Spaces and Their Mirrors", Nucl. Phys. B477 (1996) 407, hep-th/9606112. 
[31] A. Strominger, "Special Geometry", Commun. Math. Phys. 133 (1990) 163.

[32] E. Witten, "Mirror Manifolds and Topological Field Theory", in Essays on mirror manifolds, ed. S.T. Yau, Hong Kong Int. Press, 1992, pp. 120.

[33] For a review see, for example,

S. Hosono, A. Klemm and S. Theisen, "Lectures on Mirror Symmetry", hepth/9403096.

[34] G. Curio, A. Klemm, D. Lüst and S. Theisen, to appear.

[35] R. Dijkgraaf, H. Verlinde, E. Verlinde, "Topological Strings in $d<1$ ", Nucl. Phys. B352 (1991) 59.

[36] I.V. Lavrinenko, H. Lu and C.N. Pope, "From Topology to Generalised Dimensional Reduction", Nucl. Phys. B492 (1997) 278, hep-th/9611134.

[37] B. de Wit, V. Kaplunovsky, J. Louis and D. Lüst, "Perturbative Couplings of Vector Multiplets in N=2 Heterotic String Vacua", Nucl. Phys. B451 (1995) 53, hepth/9504006.

[38] C.W. Misner, K.S. Thorne and J.A. Wheeler, Gravitation, San Francisco: Freeman (1973).

[39] M. Bodner, A.C. Cadavid and S. Ferrara, "(2,2) Vacuum Configurations for Type IIA Superstrings: N=2 Supergravity Lagrangians and Algebraic Geometry", Class. Quant. Grav. 8 (1991) 789.

[40] P. Candelas and X.C. de la Ossa, "Moduli Space of Calabi-Yau Manifolds", Nucl. Phys. B355 (1991) 455. 